\title{
TU/e emonownen

\section{Combined longitudinal and lateral control of car-like vehicle platooning with extended look-ahead}

\section{Citation for published version (APA):}

Bayuwindra, A., Ploeg, J., Lefeber, E., \& Nijmeijer, H. (2020). Combined longitudinal and lateral control of carlike vehicle platooning with extended look-ahead. IEEE Transactions on Control Systems Technology, 28(3), 790-803. [8635477]. https://doi.org/10.1109/TCST.2019.2893830

\section{Document license:}

TAVERNE

DOI:

10.1109/TCST.2019.2893830

Document status and date:

Published: 01/05/2020

\section{Document Version:}

Publisher's PDF, also known as Version of Record (includes final page, issue and volume numbers)

\section{Please check the document version of this publication:}

- A submitted manuscript is the version of the article upon submission and before peer-review. There can be important differences between the submitted version and the official published version of record. People interested in the research are advised to contact the author for the final version of the publication, or visit the $\mathrm{DOI}$ to the publisher's website.

- The final author version and the galley proof are versions of the publication after peer review.

- The final published version features the final layout of the paper including the volume, issue and page numbers.

Link to publication

\section{General rights}

Copyright and moral rights for the publications made accessible in the public portal are retained by the authors and/or other copyright owners and it is a condition of accessing publications that users recognise and abide by the legal requirements associated with these rights.

- Users may download and print one copy of any publication from the public portal for the purpose of private study or research.

- You may not further distribute the material or use it for any profit-making activity or commercial gain

- You may freely distribute the URL identifying the publication in the public portal.

If the publication is distributed under the terms of Article 25fa of the Dutch Copyright Act, indicated by the "Taverne" license above, please follow below link for the End User Agreement:

www.tue.nl/taverne

Take down policy

If you believe that this document breaches copyright please contact us at:

openaccess@tue.nl

providing details and we will investigate your claim. 


\title{
Combined Longitudinal and Lateral Control of Car-Like Vehicle Platooning With Extended Look-Ahead
}

\author{
Anggera Bayuwindra $^{(}$, Jeroen Ploeg $^{(}{ }^{(}$Erjen Lefeber $^{(}$, and Henk Nijmeijer ${ }^{\circledR}$, Fellow, IEEE
}

\begin{abstract}
In this paper, we present a novel look-ahead concept for combined longitudinal and lateral vehicle following control for a car-like platoon. A nonlinear controller structure, which is based on Cooperative Adaptive Cruise Control, is designed for the lateral and longitudinal direction. For practical implementation and cost efficiency, a preceding vehicle look-ahead approach is considered since it utilizes the already available information (such as preceding vehicle position, orientation, and velocity) from radar and vehicle-to-vehicle communication. However, due to the position control in the look-ahead approach, the follower vehicle may cut corners. To overcome this problem, the lookahead is extended to a point perpendicular to the direction of the preceding vehicle, which can be viewed as a virtual preceding vehicle tracking objective. To demonstrate the effectiveness of the designed controller with the extended look-ahead approach, simulations are performed and further validated with experiments on a mobile robot platform. The results prove the effectiveness of the extended look-ahead approach.
\end{abstract}

Index Terms-Control algorithm, Cooperative Adaptive Cruise Control (CACC), intelligent vehicle, nonlinear systems, vehicle platooning.

\section{INTRODUCTION}

$\mathbf{T}$ HE increasing needs of transportation that are not balanced by the growth of highway capacity lead to traffic congestion. Hence, vehicle platooning is developed as an effective means to increase highway capacity by maintaining the intervehicle distance [1]. The concept of maintaining a desired intervehicle distance is first introduced by the invention of Adaptive Cruise Control (ACC). By means of a radar and lidar, ACC measures the distance and the relative speed of the preceding vehicle and adapts the velocity of the following vehicle in order to maintain a desired distance. As an extension to ACC, Cooperative ACC (CACC) was developed by adding vehicle-to-vehicle (V2V) communication. By providing the following vehicle with more information about its preceding

Manuscript received January 22, 2018; revised August 20, 2018; accepted December 25, 2018. Date of publication February 5, 2019; date of current version April 13, 2020. Manuscript received in final form January 15, 2019. This work was supported by the Indonesian Ministry of Finance through the Indonesia Endowment Fund for Education under Grant S-218/LPDP/2013. Recommended by Associate Editor G. Hu. (Corresponding author: Anggera Bayuwindra.)

A. Bayuwindra, E. Lefeber, and H. Nijmeijer are with the Department of Mechanical Engineering, Eindhoven University of Technology, 5600 MB Eindhoven, The Netherlands (e-mail: a.bayuwindra@tue.nl; a.a.j.lefeber@tue.nl; h.nijmeijer@tue.nl).

J. Ploeg is with 2getthere, 3543 AE Utrecht, The Netherlands, and also with Department of Mechanical Engineering, Eindhoven University of Technology, 5600 MB Eindhoven, The Netherlands (e-mail: jeroen@2getthere.eu).

Color versions of one or more of the figures in this article are available online at http://ieeexplore.ieee.org.

Digital Object Identifier 10.1109/TCST.2019.2893830 vehicle, the addition of $\mathrm{V} 2 \mathrm{~V}$ communication has been proven to reduce the intervehicle distance while attenuating disturbances in upstream direction [2], [3]. In addition, by driving closer behind one another, the aerodynamic drag force between vehicles can be reduced, especially for heavy-duty vehicles, thus resulting in lower emissions and fuel consumption of all vehicles in a platoon [4], [5].

Vehicle platooning, which can be described as a "follow the leader" strategy, is realized by exchanging information about the longitudinal and lateral properties between vehicles. To achieve a fully automated vehicle platooning, several strategies have been introduced in the literature. In [6], the longitudinal and lateral control system for automated vehicles in a platoon is introduced as independent systems. The longitudinal controller is based on CACC, with the objective of maintaining constant spacing between vehicles, while the lateral control is developed based on a lane-keeping approach. In other literature, longitudinal and lateral controllers are treated as coupled systems. The studies in [7] and [8] show that the coupling compensation improves the control performance. In general, the longitudinal controller of vehicle platooning is designed based on CACC, with an objective to maintain a desired longitudinal intervehicle spacing. On the other hand, the lateral controller can be designed either based on: lane-keeping (e.g., [6], [9], [10]) or vehicle following (e.g., [11], [12]). In [6], a lane-keeping method with a magnetic marker system is used for the lateral control system. The magnetic marker system uses magnetometers mounted on the vehicles and senses the magnetic field intensity from magnets embedded in the lane to measure the lateral distance from the center of the lane, hence earns the nickname "look-down" sensing system. Although the proposed control system had been proven to be reliable and robust, this approach is impractical because every lane needs to be embedded with magnets. In another approach [9], the lateral control is developed using a vision-based lane-keeping system. Instead of embedding magnets in lanes, this vision-based look-down system employs a camera-based image processing algorithm for lane detection. This approach is analogous to the path-tracking approach that has been widely adopted in robot motion. From a platooning viewpoint, there are several considerations in a lane-keeping method that should be taken into account. First, when the vehicles in a platoon are driving close together, it is not always possible to obtain an accurate measurement of lane markings [11]. Second, the lane markings also may be of bad quality, obstructed by dirt/snow, 
or even not present (e.g., on intersections or rural areas), upon which vehicle following can serve as a fallback.

In the situation, when the lane markings are not available, a more reliable approach is to track the preceding vehicle's lateral position based on the preceding vehicle's position and orientation (measured by the radar) and the preceding vehicle's velocity and acceleration (communicated through $\mathrm{V} 2 \mathrm{~V}$ ). Thus, a vehicle-following controller based on the look-ahead approach is introduced [13]. However, the application of this look-ahead approach for lateral behavior has some drawbacks in the event of a cornering maneuver. The look-ahead control can command the follower vehicle to turn when it senses the difference between the orientation of the preceding and the follower vehicle, causing the follower to turn too early, thus cutting the corner. The deviation in the corner radius escalates with the distance to the preceding vehicle [14]. To address the cutting-corner behavior, several approaches have been proposed. The Control Using Trajectory algorithm, introduced in [14], makes use of the time history associated with the preceding vehicle. The position coordinates of the preceding vehicle and the motion parameters of the follower vehicle are stored to determine the previous trajectory of the preceding vehicle. In [12] and [15], the cutting-corner behavior is compensated by shifting the reference point from the rear of the preceding vehicle to a static point behind it. For a platoon consisting of two vehicles, the results show that the cutting-corner behavior has been compensated for a constant velocity scenario. Most of the controllers designed in the existing research (e.g., [6], [11], [12]) heavily depend on the assumption of constant or positive velocity for all vehicles in a platoon, which results in a linear state-space model that is controlled with pole placement or optimal state feedback.

The main contribution of this paper consists of the design of an extended look-ahead approach that can compensate cutting-corner behavior in vehicle platooning, with emphasis on the cornering maneuver while maintaining a safe intervehicle distance. This extended look-ahead approach uses the velocity and heading direction information of the preceding vehicle (which are already available from radar and $\mathrm{V} 2 \mathrm{~V}$ ), to create the "virtual" vehicle as a new tracking objective. A formal stability analysis of the resulting closed-loop model is provided using a Lyapunov-based method. To guarantee the asymptotic stability and the nonzero velocity of all vehicles, we define the maximum bounds of lateral and longitudinal accelerations. Compared to the existing results of other vehicle-following controllers, our designed controllers do not depend on the assumption of constant velocity and take the motion constraints of lateral and longitudinal accelerations into account.

The remaining part of this paper is composed as follows. Section II describes the vehicle model and the lookahead-based controller design. In Section III, the extended look-ahead approach is proposed. The system is simulated in MATLAB and the results are presented in Section IV. For further validation, the designed controller is also implemented in a unicycle mobile robot platform, as presented in Section V. Section VI summarizes the conclusions.

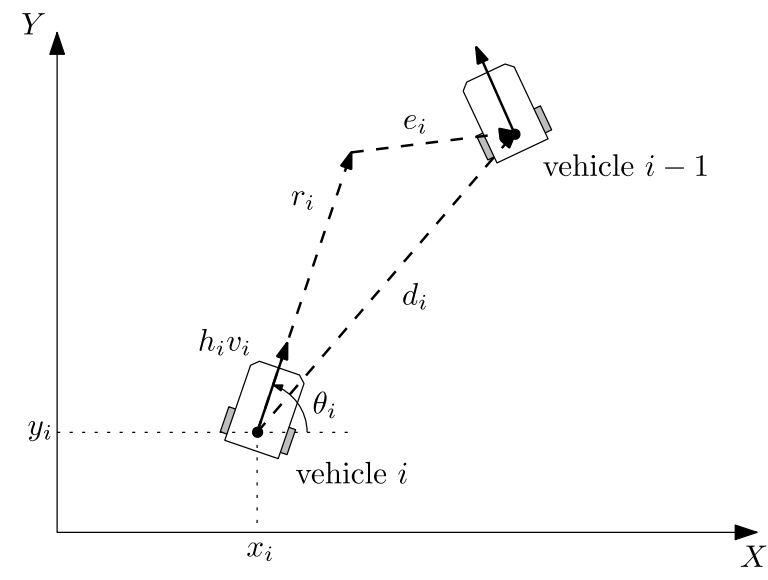

Fig. 1. Error definition in vector term for follower vehicles, where $e_{i}$ is the spacing error and $d_{i}$ is the actual distance between vehicle $i$ and $i-1$.

\section{Vehicle Modeling And Look-Ahead-BASed CONTROLler DESIGN}

The vehicle in a platoon is modeled as a unicycle on a Cartesian coordinate system. Consider a platoon of $m \in \mathbb{N}$ vehicles, with $S_{m}=\{i \in \mathbb{N} \mid 1 \leq i \leq m\}$ denoting the set of all vehicles in the platoon. The unicycle kinematic model is described by the following differential equations:

$$
\begin{aligned}
\dot{x}_{i} & =v_{i} \cos \theta_{i} \\
\dot{y}_{i} & =v_{i} \sin \theta_{i} \\
\dot{v}_{i} & =a_{i} \\
\dot{\theta}_{i} & =\omega_{i}
\end{aligned}
$$

where $\left(x_{i}, y_{i}\right)$ are the Cartesian coordinates of the unicycle, $\theta_{i}$ is the orientation of the unicycle with respect to the $x$ axis, $v_{i}$ is the longitudinal velocity, $a_{i}$ is the longitudinal acceleration input, $\omega_{i}$ is the angular velocity input, and $i \in S_{m}$ is the vehicle index, increasing in the upstream direction.

The main objective of vehicle $i$ in a platoon is to follow the preceding vehicle $i-1$ at a desired distance $d_{r, i}$. The desired distance between vehicles can be chosen as: 1) a constant spacing policy or 2) a constant time-gap spacing policy, in which the spacing policy is velocity dependent. The constant time gap spacing policy is adapted from [3] and [13], which in this paper is formulated as

$$
d_{r, i}=\left[\begin{array}{l}
d_{r x, i} \\
d_{r y, i}
\end{array}\right]=\left(r_{i}+h_{i} v_{i}\right)\left[\begin{array}{c}
\cos \theta_{i} \\
\sin \theta_{i}
\end{array}\right]
$$

where $d_{r, i}$ is the desired distance vector between vehicle $i$ and vehicle $i-1, r_{i}>0$ is the standstill distance, $h_{i}>0$ is the time gap, $v_{i}$ is the velocity of vehicle $i$, and $\theta_{i}$ is the heading angle of vehicle $i$ with respect to the $x$-axis. It should be noted that in contrast to [3] and [13], in this formulation, the standstill distance is defined as a vector with angle $\theta_{i}$ and can be understood as a look-ahead in the same direction as the vehicle's orientation (see Fig. 1). The time gap $h_{i}$ can be considered as the time needed by vehicle $i$ to reach the current position of its preceding vehicle when traveling at constant velocity $v_{i}$. 
It is assumed that each vehicle in the platoon can communicate and sense the movement of the neighboring vehicle, as depicted in Fig. 1. Let $p_{i}:=\left[x_{i}, y_{i}\right]^{T}$ be the position of vehicle $i$. Since the main focus of vehicle platooning is to maintain the intervehicle distance, it makes sense to derive the error dynamics based on the difference between the desired distance $d_{r, i}$ and the actual intervehicle distance. The spacing error vector is defined as

$$
e_{i}=d_{i}-d_{r, i}=\left(p_{i-1}-p_{i}\right)-d_{r, i}
$$

with $d_{r, i}$ as in (2), and $d_{i}=\left(d_{x, i}, d_{y, i}\right)^{T}$ is the actual distance between vehicle $i$ and vehicle $i-1$. The spacing error (3) is decomposed in a global Cartesian coordinate system as follows:

$$
\begin{aligned}
& e_{x, i}=x_{i-1}-x_{i}-\left(r_{i}+h_{i} v_{i}\right) \cos \theta_{i} \\
& e_{y, i}=y_{i-1}-y_{i}-\left(r_{i}+h_{i} v_{i}\right) \sin \theta_{i} .
\end{aligned}
$$

To proceed, we define the following state components:

$$
\begin{aligned}
& z_{1, i}=x_{i-1}-x_{i}-\left(r_{i}+h_{i} v_{i}\right) \cos \theta_{i} \\
& z_{2, i}=y_{i-1}-y_{i}-\left(r_{i}+h_{i} v_{i}\right) \sin \theta_{i} \\
& z_{3, i}=v_{i-1} \cos \theta_{i-1}-v_{i} \cos \theta_{i} \\
& z_{4, i}=v_{i-1} \sin \theta_{i-1}-v_{i} \sin \theta_{i} .
\end{aligned}
$$

It can be observed that the first and second components of (5) are identical to the position errors $(4 a, 4 b)$, while the third and fourth components represent the velocity error in the $x$ - and $y$ direction, respectively. The intervehicle dynamics is obtained by differentiating (5) with respect to time, resulting in

$$
\begin{aligned}
& {\left[\begin{array}{l}
\dot{z}_{1, i} \\
\dot{z}_{2, i}
\end{array}\right]=\left[\begin{array}{l}
z_{3, i} \\
z_{4, i}
\end{array}\right]-F_{i}\left[\begin{array}{l}
a_{i} \\
\omega_{i}
\end{array}\right]} \\
& {\left[\begin{array}{l}
\dot{z}_{3, i} \\
\dot{z}_{4, i}
\end{array}\right]=H_{i-1}\left[\begin{array}{l}
a_{i-1} \\
\omega_{i-1}
\end{array}\right]-H_{i}\left[\begin{array}{l}
a_{i} \\
\omega_{i}
\end{array}\right]}
\end{aligned}
$$

with

$$
\begin{aligned}
F_{i} & :=\left[\begin{array}{cc}
h_{i} \cos \theta_{i} & -\left(r_{i}+h_{i} v_{i}\right) \sin \theta_{i} \\
h_{i} \sin \theta_{i} & \left(r_{i}+h_{i} v_{i}\right) \cos \theta_{i}
\end{array}\right] \\
H_{j} & :=\left[\begin{array}{cc}
\cos \theta_{j}-v_{j} \sin \theta_{j} \\
\sin \theta_{j} & v_{j} \cos \theta_{j}
\end{array}\right], \quad j \in\{i-1, i\} .
\end{aligned}
$$

The system (6) represents the dynamics of the intervehicle error between vehicle $i-1$ and $i$. The objective is now to design a control input $\left[a_{i}, \omega_{i}\right]^{T}$ which asymptotically stabilizes the system (6) at zero. Let $r_{i}>0$ and $h>0$. By choosing the control input as

$$
\begin{aligned}
{\left[\begin{array}{c}
a_{i} \\
\omega_{i}
\end{array}\right] } & =F_{i}^{-1}\left[\begin{array}{l}
z_{3, i}+k_{1, i} z_{1, i} \\
z_{4, i}+k_{2, i} z_{2, i}
\end{array}\right] \\
& =\left[\begin{array}{cc}
\frac{1}{h_{i}} \cos \theta_{i} & \frac{1}{h_{i}} \sin \theta_{i} \\
-\frac{\sin \theta_{i}}{r_{i}+h_{i} v_{i}} & \frac{\cos \theta_{i}}{r_{i}+h_{i} v_{i}}
\end{array}\right]\left[\begin{array}{l}
z_{3, i}+k_{1, i} z_{1, i} \\
z_{4, i}+k_{2, i} z_{2, i}
\end{array}\right]
\end{aligned}
$$

where $r_{i}+h_{i} v_{i}>0$, the closed-loop error dynamics is obtained by substituting (8) into (6), eventually resulting in

$$
\begin{aligned}
& {\left[\begin{array}{l}
\dot{z}_{1, i} \\
\dot{z}_{2, i}
\end{array}\right]=-\left[\begin{array}{cc}
k_{1, i} & 0 \\
0 & k_{2, i}
\end{array}\right]\left[\begin{array}{l}
z_{1, i} \\
z_{2, i}
\end{array}\right]} \\
& {\left[\begin{array}{l}
\dot{z}_{3, i} \\
\dot{z}_{4, i}
\end{array}\right]=-G_{i}\left[\begin{array}{l}
z_{3, i} \\
z_{4, i}
\end{array}\right]+\left[\begin{array}{l}
\xi_{1, i} \\
\xi_{2, i}
\end{array}\right]}
\end{aligned}
$$

with

$$
\begin{aligned}
{\left[\begin{array}{l}
\xi_{1, i} \\
\xi_{2, i}
\end{array}\right]:=H_{i-1}\left[\begin{array}{l}
a_{i-1} \\
\omega_{i-1}
\end{array}\right]-G_{i}\left[\begin{array}{l}
k_{1, i} z_{1, i} \\
k_{2, i} z_{2, i}
\end{array}\right] } \\
G_{i}:=\left[\begin{array}{ll}
\frac{h_{i} v_{i}+r_{i} \cos ^{2} \theta_{i}}{h_{i}\left(r_{i}+h_{i} v_{i}\right)} & \frac{r_{i} \sin \theta_{i} \cos \theta_{i}}{h_{i}\left(r_{i}+h_{i} v_{i}\right)} \\
\frac{r_{i} \sin \theta_{i} \cos \theta_{i}}{h_{i}\left(r_{i}+h_{i} v_{i}\right)} & \frac{h_{i} v_{i}+r_{i} \sin ^{2} \theta_{i}}{h_{i}\left(r_{i}+h_{i} v_{i}\right)}
\end{array}\right]
\end{aligned}
$$

and $H_{i-1}$ as in (7b). With the controller (8), we obtained a linear system (9a) of which the poles can be place anywhere. Since the identical behavior for the $x$ - and $y$-direction is desired, we can choose the same value for $k_{1, i}=k_{1}$ and $k_{2, i}=k_{2}$ for all vehicles. Note that the input (8) requires the distance error $\left(z_{1, i}, z_{2, i}\right)$ that can be obtained using a radar, and also the preceding vehicle velocity $v_{i-1}$ and heading angle $\theta_{i-1}$, which can be obtained through wireless communication. By choosing $k_{1}, k_{2}>0$, the first subsystem (9a) is globally asymptotically stable. Consequently, $\left(z_{1, i}, z_{2, i}\right)$ converges to zero. It should also be noted that the condition of $r_{i}+h_{i} v_{i}>0$, which is sufficiently fulfiled by $v_{i}>0$ (forward driving), is necessary to determine the input (8). Thus, we establish the stability of the overall system (9) and the condition of $v_{i}>0$ by the following result.

Proposition 1: Let $z_{i}=\left[z_{1, i}, z_{2, i}, z_{3, i}, z_{4, i}\right]^{T}, z_{12, i}=$ $\left[z_{1, i}, z_{2, i}\right]^{T}$, and $z_{34, i}=\left[z_{3, i}, z_{4, i}\right]^{T}$. Consider the system (6) in closed loop with the control input (8). Assume that $0<$ $v_{i-1}^{\min } \leq v_{i-1}(t) \leq v_{i-1}^{\max }$ and let some $\epsilon>0$ be given satisfying $\epsilon<v_{i-1}^{\min }$. If

$$
\left\|H_{i-1}\left[\begin{array}{c}
a_{i-1} \\
\omega_{i-1}
\end{array}\right]\right\|_{2}=\left\|\left[\begin{array}{c}
\ddot{x}_{i-1} \\
\ddot{y}_{i-1}
\end{array}\right]\right\|_{2}<\frac{v_{i-1}^{\min }-\epsilon}{\frac{r_{i}}{\epsilon}+h_{i}}
$$

then for sufficiently small initial conditions $z_{i}(0)$ we have that $v_{i}(t)>0$ and the input (8) is well defined. Furthermore, we have $\lim _{t \rightarrow \infty}\left\|z_{12, i}(t)\right\|=0$, and $z_{34, i}(t)$ remains bounded. Additionally, if

$$
\lim _{t \rightarrow \infty} H_{i-1}\left[\begin{array}{c}
a_{i-1} \\
\omega_{i-1}
\end{array}\right]=\lim _{t \rightarrow \infty}\left[\begin{array}{c}
\ddot{x}_{i-1}(t) \\
\ddot{y}_{i-1}(t)
\end{array}\right]=0,
$$

then we have also $\lim _{t \rightarrow \infty}\left\|z_{34, i}(t)\right\|=0$, i.e., the closed-loop system (6) and (8) is asymptotically stable.

\section{Proof: See Appendix A.}

Note that the conditions (12) and (13) are related to the constraints of accelerations in $x$ and $y$, which can also be translated as constraints of longitudinal and lateral accelerations of the preceding vehicle in the platoon.

\section{Extended Look-AheAd Controller Design}

From (3), we can observe that the look-ahead design is based on the spacing policy as commonly used for CACC. From a platooning perspective, the disadvantage of this look-ahead strategy is that the follower vehicle's lateral position is only without error on a straight line. At a cornering maneuver, this disadvantage leads to a common problem, known as cutting-corner behavior [16]. Without prior knowledge of the reference trajectory, the position controller in the conventional look-ahead design can make the follower vehicles turn too early at a cornering maneuver. In the event of 


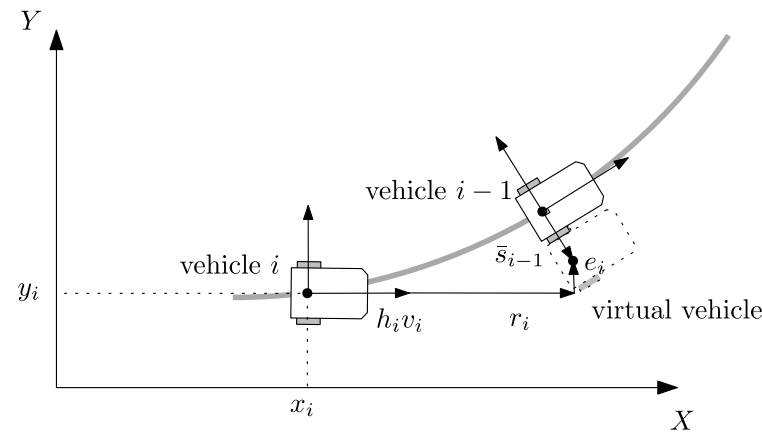

Fig. 2. Error definition for the extended look-ahead approach, where $e_{i}$ is the spacing error.

cornering, vehicle $i$ senses a position error due to the spacing policy in the conventional look-ahead approach and will turn sooner to correct errors, rather than wait until it arrives at the point of cornering. In order to make the vehicle $i$ turn at the point of the corner, the look-ahead point of the vehicle $i$ has to be extended, thus creating a "virtual" vehicle as the new tracking point for vehicle $i$. The purpose of this extension vector is to compensate the unintended lateral error due to the desired spacing distance. By using this extension vector, vehicle $i$ tracks the virtual point on vehicle $i-1$, thus allowing it to turn at the point of cornering.

Let $s_{i-1}=\left[s_{x, i-1}, s_{y, i-1}\right]^{T}$ denote the look-ahead point extending from the base of vehicle $i-1$ (Fig. 2), that can be viewed as a virtual vehicle acting as a position tracking point objective for vehicle $i$. In this approach, the look-ahead point is extended perpendicular to the heading direction of vehicle $i-1$. This extended look-ahead is applied during cornering maneuvers, i.e., when $\omega_{i-1} \neq 0$. Using the new tracking point objective, the spacing error for the extended look-ahead CACC is defined as

$$
e_{i}=\left(p_{i-1}+s_{i-1}\right)-\left(p_{i}+r_{i}+h_{i} v_{i}\right) .
$$

The components of $s_{i-1}$ in Cartesian coordinates are derived as follows:

$$
\begin{aligned}
& s_{x, i-1}=\bar{s}_{i-1} \sin \theta_{i-1} \\
& s_{y, i-1}=-\bar{s}_{i-1} \cos \theta_{i-1}
\end{aligned}
$$

where $\bar{s}_{i-1}$ is the magnitude of the extension vector $s_{i-1}$. To prevent cutting corners, our aim is to formulate the extension vector $s_{i-1}$ such that the vehicle $i$ has the same turning radius as its preceding vehicle $i-1$. Since the direction of $s_{i-1}$ is always perpendicular to the heading direction of vehicle $i-1$, only the magnitude (denoted by $\bar{s}_{i-1}$ ) needs to be determined [16]. The definition of $\bar{s}_{i-1}$ can be made by analyzing the steady-state behavior of the platoon on the circular movement, i.e., when errors $e_{x, i}$ and $e_{y, i}$ equal zero. In this approach, it is assumed that the heading angle difference between the preceding and follower vehicle is always smaller than $\pi / 2$ such that the extension vector can always be defined. When vehicle $i-1$ turns to the positive $y$-axis, it will have a turning radius $R_{i-1}$ and the new tracking point of vehicle $i$ is extended to the point $S$ (see Fig. 3). It therefore follows that:

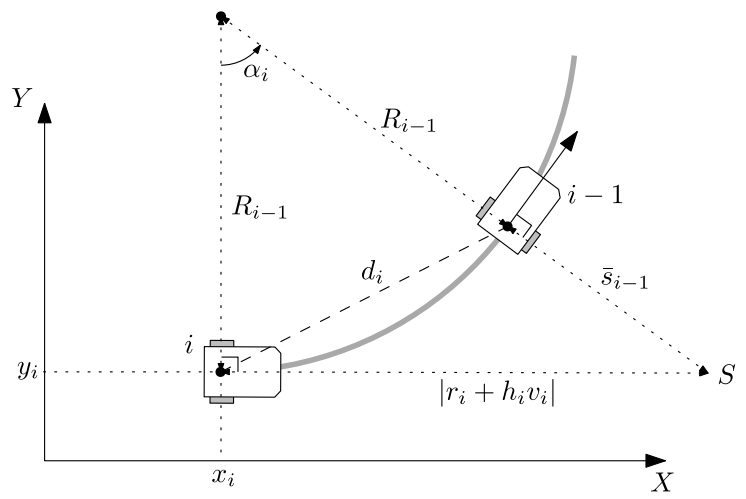

Fig. 3. Magnitude of the extension vector $\bar{s}_{i-1}$, where $d_{i}$ is the actual distance between vehicle $i$ and $i-1$.

$$
\left(R_{i-1}+\bar{s}_{i-1}\right)^{2}=R_{i-1}^{2}+\left(r_{i}+h_{i} v_{i}\right)^{2},
$$

with $R_{i-1}$ as the turning radius of vehicle $i-1, \bar{s}_{i-1}$ as the magnitude of the extension vector, and $\left(r_{i}+h_{i} v_{i}\right)$ as the magnitude of the spacing policy. Let us define $\kappa_{i-1}$ as the path curvature of vehicle $i-1$. By noting the fact that $\kappa_{i-1}=1 / R_{i-1}=\omega_{i-1} / v_{i-1}$ and by rearranging terms in (17), we define the magnitude of the extension vector $\bar{s}_{i-1}$ as

$$
\bar{s}_{i-1}= \begin{cases}0 & \text { for } \kappa_{i-1}=0 \\ \frac{-1+\sqrt{1+\kappa_{i-1}^{2}\left(r_{i}+h_{i} v_{i}\right)^{2}}}{\kappa_{i-1}} & \text { for } \kappa_{i-1} \neq 0 .\end{cases}
$$

For the case of $\kappa_{i-1}=0$, which implies that $\omega_{i-1}=0$, we have the condition in which the vehicle $i-1$ drives on a straight line. Thus, $\bar{s}_{i-1}$ is zero and the definition of spacing error in (14) will be identical to (1). To show that $\bar{s}_{i-1}$ is continuous at $\kappa_{i-1}=0$, we can use a Taylor expansion and take the limit as $\kappa_{i-1} \rightarrow 0$, such that

$$
\lim _{\kappa_{i-1} \rightarrow 0} \bar{s}_{i-1}=\lim _{\kappa_{i-1} \rightarrow 0}\left(\frac{1}{2} \kappa_{i-1}\left(r_{i}+h_{i} v_{i}\right)^{2}+\cdots\right)=0 .
$$

In Section II, the new coordinates $z_{3, i}$ and $z_{4, i}$ can be regarded as differences between the velocity of the preceding and follower vehicle in the $x$ - and $y$-direction, respectively, and it has been proven that the designed controller (8) asymptotically stabilizes the dynamics of $z_{3, i}$ and $z_{4, i}$. The convergence of $z_{3, i}$ and $z_{4, i}$ to zero has been shown in Section II, under the condition that $\left[a_{i-1}, \omega_{i-1}\right]^{T}$ also converges to zero. However, if a platoon maneuvers on a curved path (i.e., $\omega_{i-1}$ is nonzero), $z_{3, i}$ and $z_{4, i}$ will not converge to zero according to (9b). Therefore, we redefine the coordinate transformation of $z_{3, i}$ and $z_{4, i}$ such that the convergence of $z_{3, i}$ and $z_{4, i}$ to zero on a curved path is also guaranteed, i.e., the velocity of the follower vehicle converges to the velocity of its preceding vehicle. To proceed, we define the new state components as

$$
\begin{aligned}
& z_{1, i}=x_{i-1}+s_{x, i-1}-x_{i}-\left(r_{i}+h_{i} v_{i}\right) \cos \theta_{i} \\
& z_{2, i}=y_{i-1}+s_{y, i-1}-y_{i}-\left(r_{i}+h_{i} v_{i}\right) \sin \theta_{i} \\
& z_{3, i}=v_{i-1} \cos \theta_{i-1}-v_{i} \cos \left(\theta_{i}+\alpha_{i}\right) \\
& z_{4, i}=v_{i-1} \sin \theta_{i-1}-v_{i} \sin \left(\theta_{i}+\alpha_{i}\right) .
\end{aligned}
$$


With this new choice of state components, the control objective can be formulated such that $\left[z_{1, i}, z_{2, i}\right]^{T}$ converges to zero. From Fig. 3, we can define $\alpha_{i}$, the angle of the arc between the vehicle $i$ and vehicle $i-1$, as a function of $\kappa_{i-1}$ and $v_{i}$ satisfying

$$
\alpha_{i}=\arctan \left[\kappa_{i-1}\left(r_{i}+h_{i} v_{i}\right)\right], \quad-\frac{\pi}{2}<\alpha_{i}<\frac{\pi}{2} .
$$

Note that the definition of $\alpha_{i}$ only applies when $\alpha_{i} \in$ $(-\pi / 2, \pi / 2)$, i.e., the orientation difference between the follower and its preceding vehicle has to be smaller than $\pi / 2$. By noting that

$$
\begin{aligned}
\sin \alpha_{i} & =\frac{\kappa_{i-1}\left(r_{i}+h_{i} v_{i}\right)}{\sqrt{1+\kappa_{i-1}^{2}\left(r_{i}+h_{i} v_{i}\right)^{2}}} \\
\cos \alpha_{i} & =\frac{1}{\sqrt{1+\kappa_{i-1}^{2}\left(r_{i}+h_{i} v_{i}\right)^{2}}}
\end{aligned}
$$

derivatives of $\alpha_{i}$ and $\bar{s}_{i-1}$ with respect to time are given as

$$
\begin{aligned}
\dot{\alpha}_{i} & =\alpha_{\kappa, i} \dot{\kappa}_{i-1}+\alpha_{a, i} a_{i} \\
\dot{\bar{s}}_{i-1} & =s_{\kappa, i} \dot{\kappa}_{i-1}+s_{a, i} a_{i}
\end{aligned}
$$

with

$$
\begin{aligned}
\alpha_{\kappa, i} & :=\left(r_{i}+h_{i} v_{i}\right) \cos ^{2} \alpha_{i}, \quad \alpha_{a, i}:=h_{i} \kappa_{i-1} \cos ^{2} \alpha_{i} \\
s_{\kappa, i} & :=\frac{1}{\kappa_{i-1}^{2}}\left(1-\cos \alpha_{i}\right), \quad s_{a, i}:=h_{i} \sin \alpha_{i} .
\end{aligned}
$$

To obtain the intervehicle dynamics in the new coordinates (24), we first define a rotation matrix

$$
R\left(\alpha_{i}\right)=\left[\begin{array}{cc}
\cos \alpha_{i} & -\sin \alpha_{i} \\
\sin \alpha_{i} & \cos \alpha_{i}
\end{array}\right]
$$

such that the term $\left[z_{3, i}, z_{4, i}\right]^{T}$ can be rewritten as

$$
\begin{aligned}
\frac{1}{\cos \alpha_{i}}\left[\begin{array}{c}
z_{3, i} \\
z_{4, i}
\end{array}\right]= & {\left[\begin{array}{c}
\cos \theta_{i-1} \\
\sin \theta_{i-1}
\end{array}\right] \frac{v_{i-1}}{\cos \alpha_{i}}-\frac{R\left(\alpha_{i}\right)}{\cos \alpha_{i}}\left[\begin{array}{c}
\cos \theta_{i} \\
\sin \theta_{i}
\end{array}\right] v_{i} } \\
= & {\left[\begin{array}{c}
\cos \theta_{i-1} \\
\sin \theta_{i-1}
\end{array}\right] \frac{v_{i-1}}{\cos \alpha_{i}}-\left[\begin{array}{c}
\cos \theta_{i} \\
\sin \theta_{i}
\end{array}\right] v_{i} } \\
& -\left[\begin{array}{c}
-\sin \theta_{i} \\
\cos \theta_{i}
\end{array}\right] v_{i} \tan \alpha_{i}
\end{aligned}
$$

By differentiating (19) with respect to time and taking equations (23), (24), and (28) into account, we eventually obtain the intervehicle dynamics as follows:

$$
\begin{aligned}
& {\left[\begin{array}{l}
\dot{z}_{1, i} \\
\dot{z}_{2, i}
\end{array}\right]=\frac{1}{\cos \alpha_{i}}\left[\begin{array}{l}
z_{3, i} \\
z_{4, i}
\end{array}\right]-\Gamma_{12, i}\left[\begin{array}{c}
a_{i} \\
\omega_{i}
\end{array}\right]+\beta_{1, i}} \\
& {\left[\begin{array}{l}
\dot{z}_{3, i} \\
\dot{z}_{4, i}
\end{array}\right]=H_{i-1}\left[\begin{array}{l}
a_{i-1} \\
\omega_{i-1}
\end{array}\right]-\Gamma_{34, i}\left[\begin{array}{c}
a_{i} \\
\omega_{i}
\end{array}\right]+\beta_{2, i}}
\end{aligned}
$$

with

$$
\begin{aligned}
& \Gamma_{12, i}=\left[\begin{array}{cc}
h_{i} \cos \theta_{i}-s_{a, i} \sin \theta_{i-1} & -\left(r_{i}+h_{i} v_{i}\right) \sin \theta_{i} \\
h_{i} \sin \theta_{i}+s_{a, i} \cos \theta_{i-1} & \left(r_{i}+h_{i} v_{i}\right) \cos \theta_{i}
\end{array}\right] \\
& \Gamma_{34 . i}=\left[\begin{array}{cc}
\cos \left(\theta_{i}+\alpha_{i}\right)-\delta_{i} \sin \left(\theta_{i}+\alpha_{i}\right)-v_{i} \sin \left(\theta_{i}+\alpha_{i}\right) \\
\sin \left(\theta_{i}+\alpha_{i}\right)+\delta_{i} \cos \left(\theta_{i}+\alpha_{i}\right) & v_{i} \cos \left(\theta_{i}+\alpha_{i}\right)
\end{array}\right] \\
& \beta_{1, i}=\left[\begin{array}{c}
-\sin \theta_{i} \\
\cos \theta_{i}
\end{array}\right] v_{i} \tan \alpha_{i}+R\left(\theta_{i-1}\right)\left[\begin{array}{c}
\bar{s}_{i-1} \omega_{i-1} \\
-s_{\kappa, i} \dot{\kappa}_{i-1}
\end{array}\right] \\
& +\left(1-\frac{1}{\cos \alpha_{i}}\right)\left[\begin{array}{c}
\cos \theta_{i-1} \\
\sin \theta_{i-1}
\end{array}\right] v_{i-1} \\
& \beta_{2, i}=v_{i}\left(r_{i}+h_{i} v_{i}\right) \cos ^{2} \alpha_{i}\left[\begin{array}{c}
\sin \left(\theta_{i}+\alpha_{i}\right) \\
-\cos \left(\theta_{i}+\alpha_{i}\right)
\end{array}\right] \dot{\kappa}_{i-1} \\
& \delta_{i}=v_{i} h_{i} \kappa_{i-1} \cos ^{2} \alpha_{i}
\end{aligned}
$$

$\bar{s}_{i-1}$ as in (18) and $H_{j}$, with $j \in\{i-1, i\}$, as in (7b). The objective is to design control inputs $\left[a_{i}, \omega_{i}\right]^{T}$ which asymptotically stabilize the states $\left[z_{1, i}, z_{2, i}, z_{3, i}, z_{4, i}\right]^{T}$ at zero. By taking the feedback as

$$
\begin{aligned}
& {\left[\begin{array}{l}
a_{i} \\
\omega_{i}
\end{array}\right]=\Gamma_{12, i}^{-1}\left(\left[\begin{array}{l}
k_{1} z_{1, i} \\
k_{2} z_{2, i}
\end{array}\right]+\frac{1}{\cos \alpha_{i}}\left[\begin{array}{l}
z_{3, i} \\
z_{4, i}
\end{array}\right]+\beta_{1, i}\right)} \\
& \Gamma_{12, i}^{-1}=\frac{1}{\mu_{i}}\left[\begin{array}{cc}
\left(r_{i}+h_{i} v_{i}\right) \cos \theta_{i} & \left(r_{i}+h_{i} v_{i}\right) \sin \theta_{i} \\
-h_{i} \sin \theta_{i}-s_{a, i} \cos \theta_{i-1} & h_{i} \cos \theta_{i}-s_{a, i} \sin \theta_{i-1}
\end{array}\right] \\
& \mu_{i}=h_{i}\left(r_{i}+h_{i} v_{i}\right)\left(1-\sin \alpha_{i} \sin \left(\theta_{i-1}-\theta_{i}\right)\right)
\end{aligned}
$$

where $r_{i}+h_{i} v_{i}>0$, and substituting (35) into (29a) and (29b), we obtain the closed-loop system as follows:

$$
\begin{aligned}
{\left[\begin{array}{l}
\dot{z}_{1, i} \\
\dot{z}_{2, i}
\end{array}\right]=} & -\left[\begin{array}{l}
k_{1} z_{1, i} \\
k_{2} z_{2, i}
\end{array}\right] \\
{\left[\begin{array}{l}
\dot{z}_{3, i} \\
\dot{z}_{4, i}
\end{array}\right]=} & H_{i-1}\left[\begin{array}{l}
a_{i-1} \\
\omega_{i-1}
\end{array}\right]+\beta_{2, i}-\Gamma_{34, i} \Gamma_{12, i}^{-1}\left[\begin{array}{l}
k_{1} z_{1, i} \\
k_{2} z_{2, i}
\end{array}\right] \\
& -\Gamma_{34, i} \Gamma_{12, i}^{-1}\left(\frac{1}{\cos \alpha_{i}}\left[\begin{array}{l}
z_{3, i} \\
z_{4, i}
\end{array}\right]+\beta_{1, i}\right) .
\end{aligned}
$$

Using controller (35), we obtain a linear system (38a), of which the desired behavior can be obtained through pole placement and many other controller design methods. It can be observed that (38a) describes the controlled dynamics and (38b) represents the internal dynamics of the system. By the choice of $k_{1}, k_{2}>0$, the dynamics (38a) is exponentially stable. Thus, we establish the stability of the overall system (38) by the following proposition, which can be regarded as the main result of this paper.

Proposition 2: Consider the system (29) in closed loop with the control input (35). Assume that $0<v_{i-1}^{\min } \leq v_{i-1}(t) \leq v_{i-1}^{\max }$ and let some $\epsilon>0$ be given such that $\epsilon<v_{i-1}^{\min }$. If

$$
\left|\kappa_{i-1}\right| \leq \frac{1}{r_{i}+h_{i} \sqrt{2}\left(v_{i-1}^{\max }+v_{i-1}^{\min }-\epsilon\right)}
$$

and

$$
h_{\kappa, i}^{\max }\left|\dot{\kappa}_{i-1}\right|+\left|a_{i-1}\right| \leq \frac{v_{i-1}^{\min }-\epsilon}{2\left(\frac{r_{i}}{\epsilon}+h_{i}\right)}
$$

with $h_{\kappa, i}^{\max }=\left(r_{i}^{2} / h_{i}\right)+h_{i} \sqrt{2}\left(v_{i-1}^{\max }+v_{i-1}^{\min }-\epsilon\right)$, then for sufficiently small initial conditions $z_{i}(0)$ we have that $v_{i}(t)>$ 0 such that input (35) is well defined. Furthermore, we have 

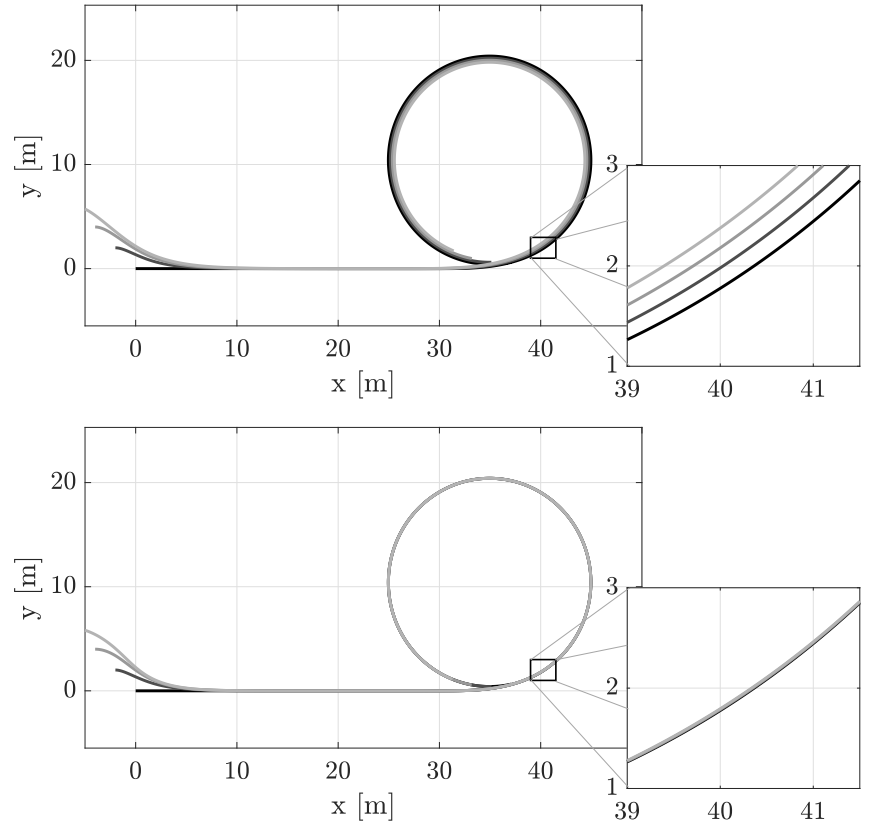

Fig. 4. Trajectory of vehicle $i$ (black-light gray: $i=1,2,3,4$ ) for the circular path scenario: conventional look-ahead (top) versus extended look-ahead (bottom).

$\lim _{t \rightarrow \infty}\left\|z_{12, i}(t)\right\|=0$, and $z_{34, i}(t)$ remains bounded. In addition, if $\lim _{t \rightarrow \infty} a_{i-1}(t)=0$ and $\dot{\kappa}_{i-1}$ converges to zero as $t \rightarrow \infty$, then we have also $\lim _{t \rightarrow \infty}\left\|z_{34, i}(t)\right\|=0$, i.e., the closed-loop system (29) and (35) is asymptotically stable.

Proof: See Appendix B.

Therefore, we can conclude that the system (38) is internally stable as long as the curvature is not too large [bounded by (39)], and (40) is satisfied. It should be noted that condition (39) is related to the bound of longitudinal and lateral velocity of the preceding vehicle, while the condition (40) is related to the lateral jerk and longitudinal acceleration of the preceding vehicle.

\section{Simulation Results}

To illustrate the effectiveness of the extended look-ahead controller, we conduct several simulations in MATLAB for a platoon of four vehicles under two scenarios: a circular path and an eight-shaped path. The spacing policy parameters are chosen as $r_{i}=1 \mathrm{~m}$ and $h_{i}=0.2 \mathrm{~s}$, and the control parameters $k_{1}=3.5$ and $k_{2}=3.5$ are selected. The gains $k_{1}$ and $k_{2}$ for the controllers are determined by an iterative manner. High value gains result in a faster convergence toward a reference path, but a higher sensitivity to curvature changes. A trajectory tracking controller is applied in the first vehicle to track the predefined trajectory, while the extended look-ahead controller is applied in other vehicles to track their preceding vehicle. It is assumed that the position, velocity, and acceleration of the preceding vehicle can be obtained without any delay involved. Both the conventional and the extended look-ahead approach are simulated for comparison.

\section{A. Circular Path Trajectory}

In this scenario, the first vehicle drives on a straight path along the $x$-axis with a constant initial velocity $v_{1}=5 \mathrm{~m} / \mathrm{s}$.
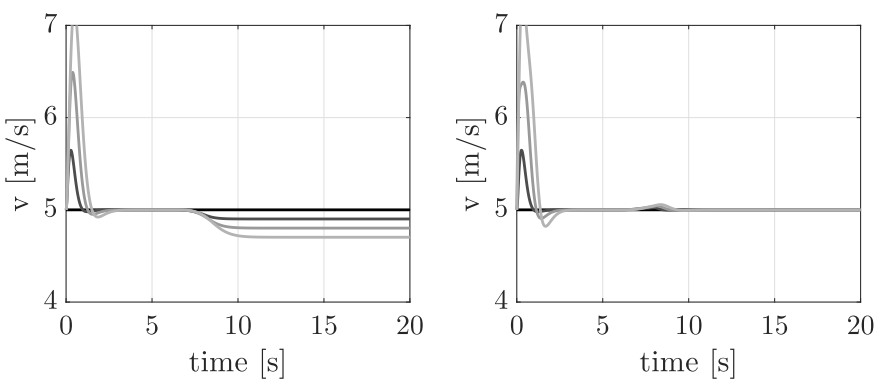

Fig. 5. Velocity of vehicle $i$ (black-light gray: $i=1,2,3,4)$ for the circular path scenario: conventional look-ahead (left) versus extended look-ahead (right).
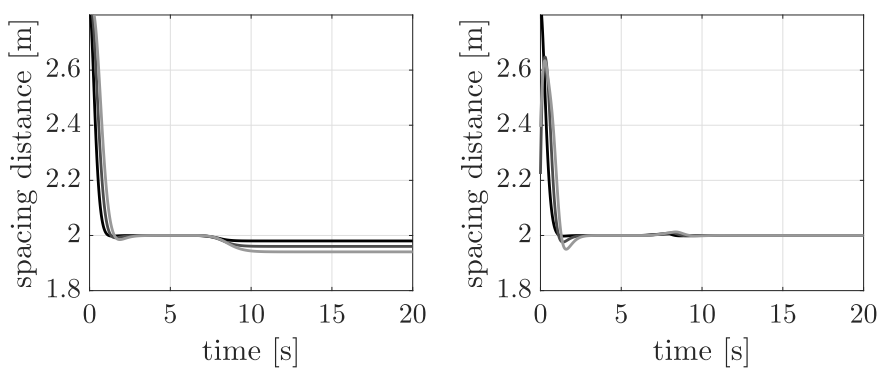

Fig. 6. Longitudinal spacing distance of vehicle $i-1$ and vehicle $i$ (blacklight gray: $i=2,3,4$ ) for the circular path scenario: conventional look-ahead (left) versus extended look-ahead (right).

At time $t=6 \mathrm{~s}$, it turns with yaw rate $\omega_{1}=0.5 \mathrm{rad} / \mathrm{s}$ and moves in a circular motion. The initial positions $\left(x_{i}(0), y_{i}(0)\right)$ are $(0,0),(-2,2),(-4,4)$, and $(-6,6)$ for vehicle $1,2,3$, and 4 , respectively, while the initial heading angle is $\theta_{i}(0)=0$ for all $i$. As depicted in Fig. 4, both look-ahead controllers perform well in the straight path, driving all follower vehicles to track their preceding vehicle without lateral error. However, the conventional look-ahead controller shows its shortcoming when the platoon enters a circular path. The vehicle with the conventional look-ahead cruises in a smaller radius than its preceding vehicle, as shown in Fig. 4. This behavior occurs because at the moment the preceding vehicle turns, the controller senses a position error and then adjusts the position of follower instantaneously. As a result, the follower vehicle turns too early and cuts the corner. From Fig. 4 (left), which shows a close-up of the circular part of the track, it can be observed that vehicles 2-4 do not travel on the path with the same radii as vehicle 1 . The difference of curvatures results in a lower longitudinal velocity of the follower vehicle than the one of the preceding vehicle, as depicted in Fig. 5. This difference in velocity also affects the longitudinal spacing distance between vehicles (Fig. 6). On the other hand, the extended look-ahead controllers successfully compensated the lateral deviation, thus keeping the follower vehicles to drive with the same radii as their preceding vehicle. By maintaining the same velocity for all vehicles, the extended look-ahead controllers manage to keep the same longitudinal spacing distance between vehicles [Fig. 6 (right)]. It is also worth noting that the velocity of all vehicles is always positive and never reaches zero for both look-ahead approaches (see Fig. 5). 

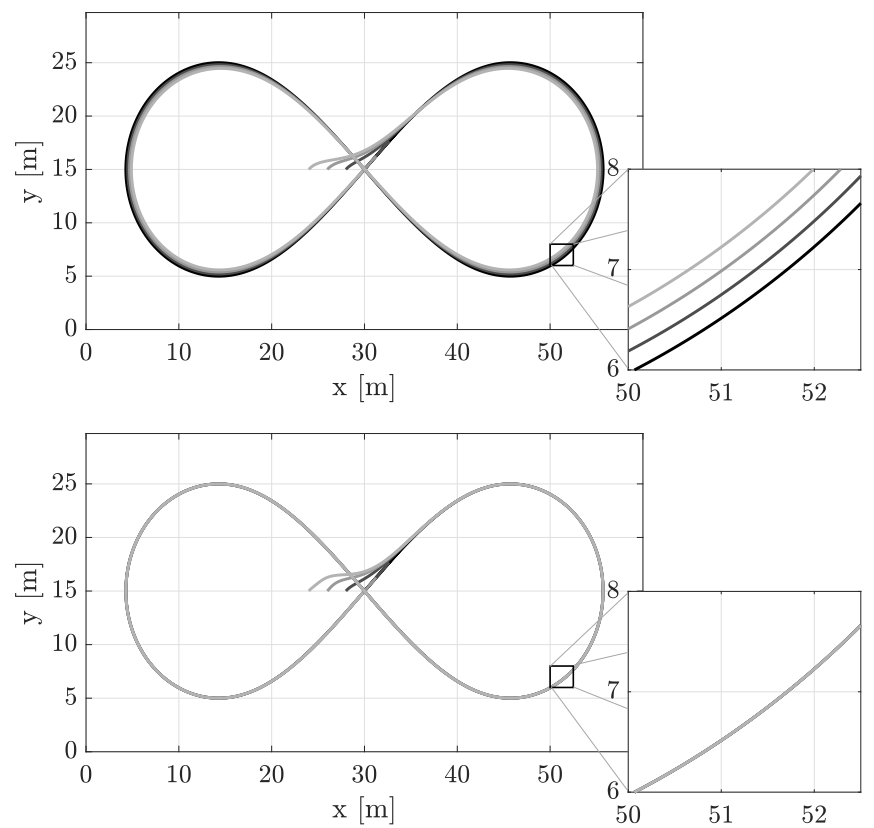

Fig. 7. Trajectory of vehicle $i$ (black-light gray: $i=1,2,3,4$ ) for the eight-shaped scenario: conventional look-ahead (top) versus extended look-ahead (bottom).
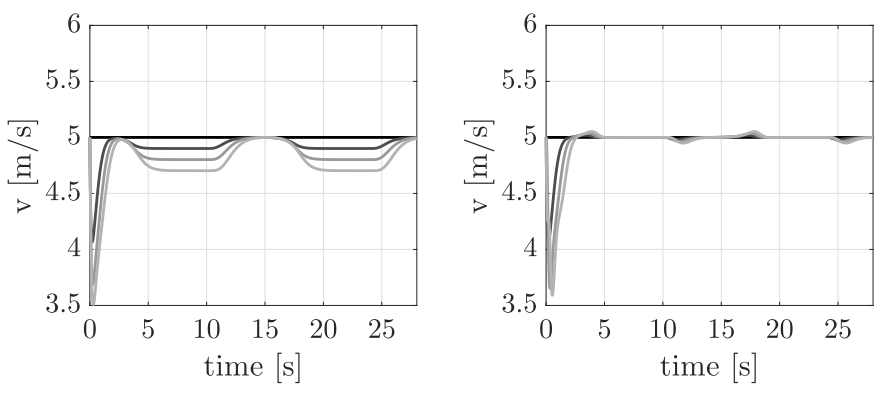

Fig. 8. Velocity of vehicle $i$ (black-light gray: $i=1,2,3,4)$ for the eight-shaped path scenario: conventional look-ahead (left) vs extended look-ahead (right).
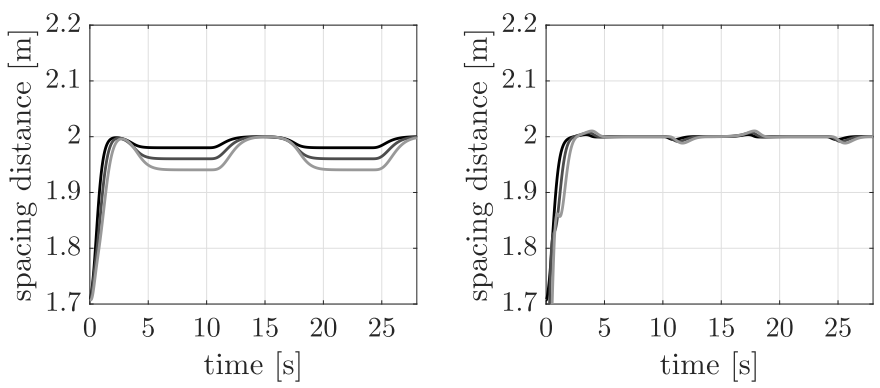

Fig. 9. Longitudinal spacing distance of vehicle $i-1$ and vehicle $i$ (blacklight gray: $i=2,3,4)$ for the eight-shaped circular path scenario: conventional look-ahead (left) versus extended look-ahead (right).

\section{B. Eight-Shaped Path Scenario}

In this scenario, the robustness of the proposed controller against varying yaw rate $\omega$ is evaluated. The first vehicle starts at initial position $(30,15)$, cruises along the eight-shaped path with a constant velocity $v_{1}=5 \mathrm{~m} / \mathrm{s}$ (equal to $18 \mathrm{~km} / \mathrm{h}$ ). The eight-shaped path is generated by half circles and quintic polynomial functions (see Fig. 7). The initial positions of the other vehicles are $(28,15),(26,15)$, and $(24,15)$ for vehicle 2 ,

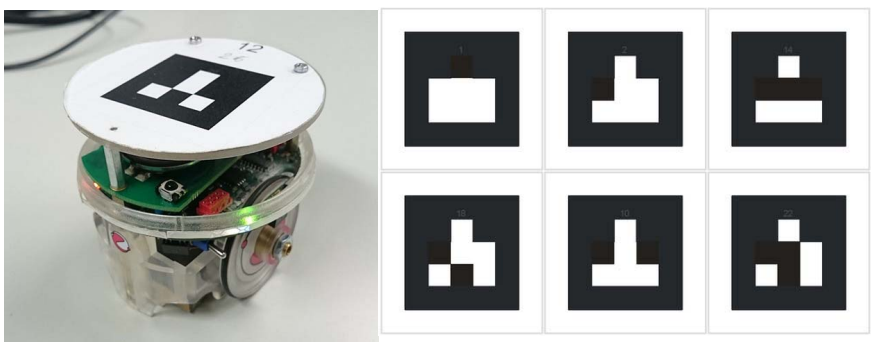

Fig. 10. E-puck mobile robot (left) and markers used for identification (right).

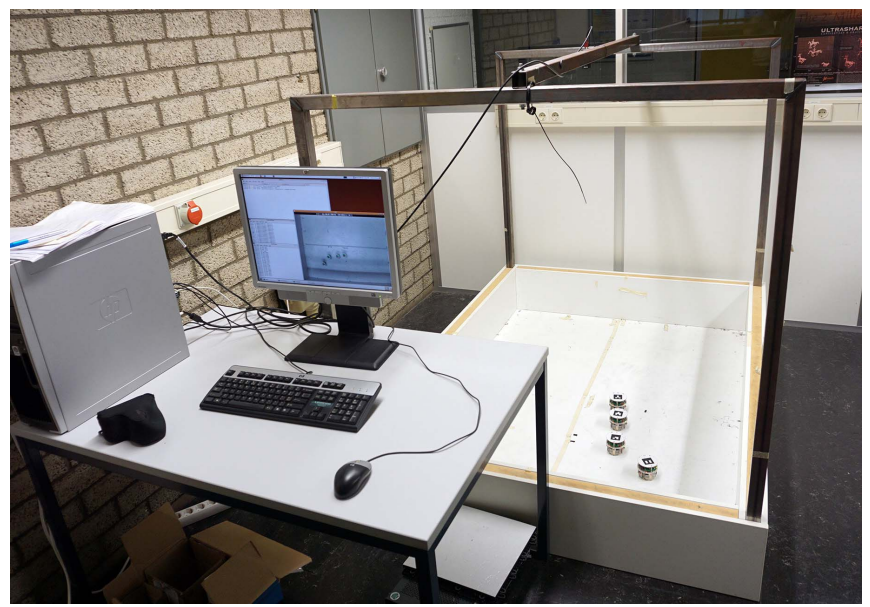

Fig. 11. Experimental setup.

3 , and 4 , respectively. The initial heading angle is $\theta_{i}(0)=$ $0.7854 \mathrm{rad}$ for all $i$. It can be observed that the initial position errors are handled equally good by both types of look-ahead controllers. However, the difference in performance can be seen in a curve path. Analogously to the simulation results of the circular path, the extended look-ahead controller delivers better tracking performance than the conventional look-ahead controller, as shown in Fig. 7. Vehicles with the conventional look-ahead drive with smaller radii than their preceding vehicle, while ones with the extended look-ahead drive with relatively equal radii with their preceding vehicle. It can also be observed from Fig. 9 (right) that the extended look-ahead controllers are capable to maintain the longitudinal spacing distance, and proven to be robust against varying yaw rate. From Fig. 8, we can observe that velocities for all vehicles are always positive and never reach zero, which is a necessary condition for the designed controllers.

\section{EXPERIMENTS}

In this section, the error dynamics and the controllers that have been derived previously are implemented using the mobile robot E-puck. E-puck is used because the implementation is straightforward, using the camera localization system developed in [17] that acquires the accurate position of unicycles. E-puck is a differential-wheeled nonholonomic mobile robot, designed by the ASL Laboratory, École Polytechnique Fédérale de Lausanne, Lausanne, Switzerland [18]. The main specifications of the E-puck robot are given in Table I. 


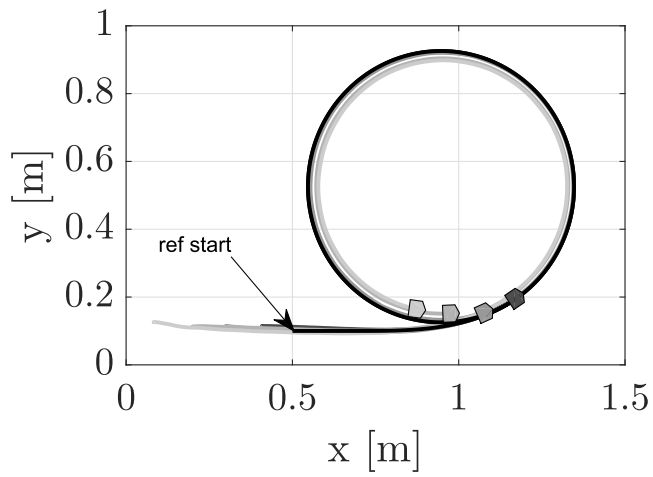

Fig. 12. Trajectory of E-puck $i$ (black-light gray: $i=1,2,3,4$ ) on a circular path with conventional look-ahead.

TABLE I

E-PUCK SPECIFICATION

\begin{tabular}{|c|c|}
\hline Specification & Values \\
\hline \hline Robot radius & $37 \mathrm{~mm}$ \\
\hline Wheel radius $(r)$ & $20.5 \mathrm{~mm}$ \\
\hline Axle length $(L)$ & $52 \mathrm{~mm}$ \\
\hline Speed unit & $0.00628 \mathrm{rad} / \mathrm{s}$ \\
\hline Encoder resolution & 159.23 \\
\hline Maximum angular speed & $1000 \mathrm{units}$ \\
\hline Maximum velocity & $13 \mathrm{~cm} / \mathrm{s}$ \\
\hline Maximum angular velocity & $5 \mathrm{rad} / \mathrm{s}$ \\
\hline
\end{tabular}

\section{A. Experiment Setup}

The experimental setup is shown in Fig. 11. Each wheel of the E-puck robot is driven by a stepper motor, and the velocity control commands are sent to both stepper motors from an external PC through Bluetooth. To be able to communicate via Bluetooth, the E-puck has to be flashed through the Bluetooth Serial Communication (SerCom) protocol that allows it to read and parse commands that are sent from the external PC. Since an E-puck is equipped with an open Bluetooth protocol, a variety of programing languages can be used to control the robot, such as through the hyperterminal of Windows, MATLAB, or Python. An external PC is used to generate a reference trajectory and to determine the absolute coordinates and orientations of the robots from a measurement device (a Firewire camera AVT Guppy F-080b b/w combined with reacTIVision software) placed above the arena of $175 \mathrm{~cm} \times 128 \mathrm{~cm}$. The position and orientation accuracy of these position measurements are $\pm 0.0019 \mathrm{~m}$ for $x$ and $y$ position and $\pm 0.0524 \mathrm{rad}$ in $\theta$ direction, with a sample rate of $25 \mathrm{~Hz}$ [19]. The PC then uses the obtained information to implement the control algorithm for each E-puck. Each E-puck is attached with a unique marker (2-D barcode) consisting of $3 \times 3$ blocks. The color of the blocks in the corners is chosen such that the orientation of the marker (thus, the orientation of the E-puck) can be determined [17], [20]. An example of the barcode used in the E-puck is shown in Fig. 10 (right). In our approach, we need to be able to measure the position of the preceding vehicle with respect to the coordinate frame of the ego vehicle. In the E-puck setup, all measurement regarding the position and orientation are done by a central camera

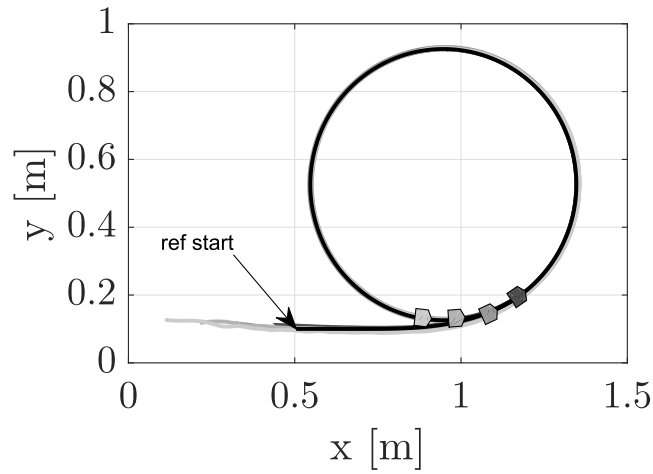

Fig. 13. Trajectory of E-puck $i$ (black-light gray: $i=1,2,3,4$ ) on a circular path with extended look-ahead.
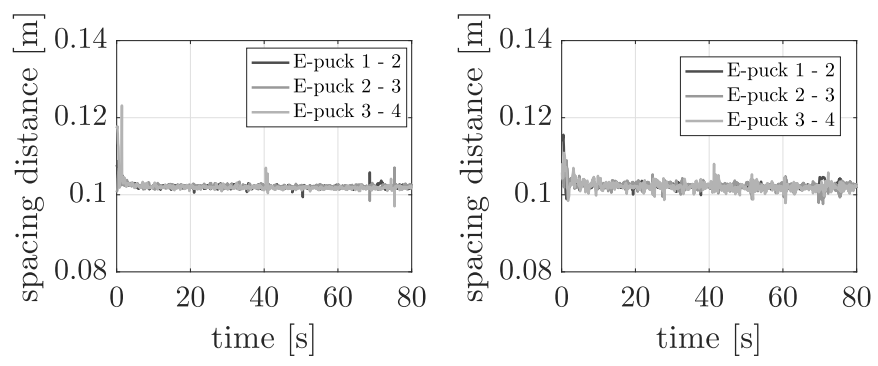

Fig. 14. Longitudinal spacing distance for the circular path scenario: conventional look-ahead (left) versus extended look-ahead (right).

placed above the arena. However, this central camera would not be available for the practical implementation in a real vehicle platooning. To address this issue, we could replace the central camera by a radar (to measure the longitudinal position of the preceding vehicle), and a forward-looking camera (to measure the lateral position), both attached on every vehicle in the platoon.

Since an E-puck is a differential-drive mobile robot, the direction of motion is controlled by separately controlling the speed of the left and right wheels, denoted by $v_{l, i}$ and $v_{r, i}$, respectively. The transformation of the individual wheels speed to the linear velocity $v_{i}$ and angular velocity $\omega_{i}$ can be determined using the following relation:

$$
\omega_{i}=\frac{v_{r, i}-v_{l, i}}{L}, \quad v_{i}=\frac{v_{r, i}+v_{l, i}}{2}
$$

where $L$ is the length between the left and right wheels.

In order to support the simulation results presented in the previous section, for this experiment, we define two kinds of reference trajectories: a circular trajectory and an eight-shaped trajectory. Given the size of the arena, an appropriate selection of the radius of the circular path is $0.4 \mathrm{~m}$ with the center point at $(0.7,0.5)$, so that the reference trajectory stays within the arena. The eight-shaped trajectory is composed of half circles with a radius of $0.3 \mathrm{~m}$ and straight sections. The reference linear velocity is chosen as $0.04 \mathrm{~m} / \mathrm{s}$, and the controlled velocities $v_{i}$ and $\omega_{i}$ of all E-pucks are physically limited by their respective maximum value as in Table I. First, the reference trajectory for the first E-puck is defined. Afterward, the positions of all E-pucks are measured and recorded in the PC through the camera. The decentralized controllers then 

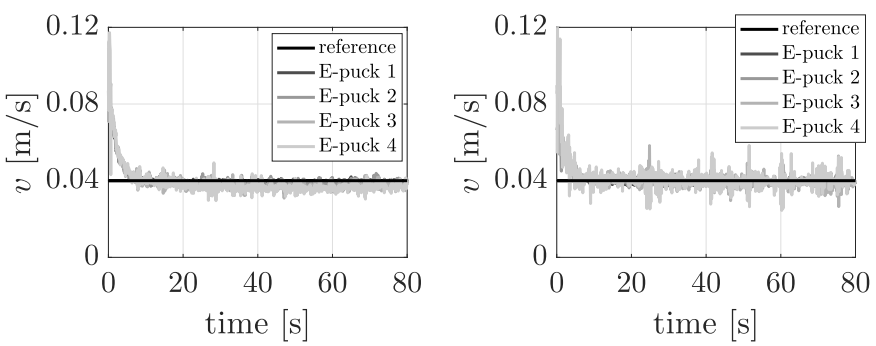

Fig. 15. Velocities of E-pucks for circular path scenario: conventional look-ahead (left) versus extended look-ahead (right).

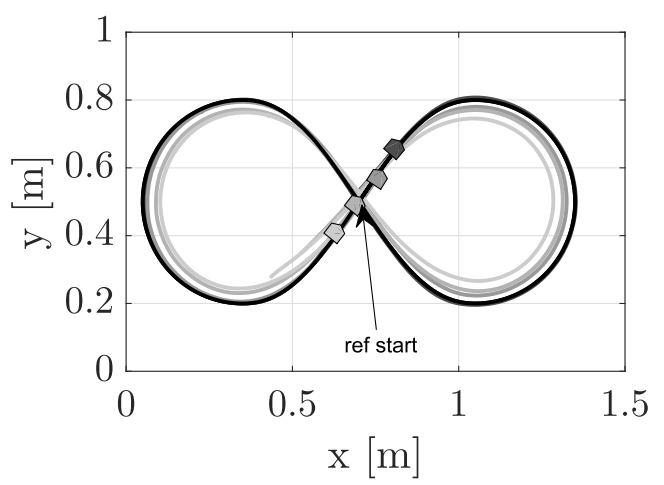

Fig. 16. Trajectory of E-puck $i$ (black-light gray: $i=1,2,3,4$ ) on an eight-shaped path with conventional look-ahead.

calculate the inputs needed for each E-puck simultaneously and send the input velocity commands via Bluetooth. It should be noted that the controllers are executed in the main PC, not in the E-pucks themselves. The control parameters are selected as $k_{1}=2$ and $k_{2}=2$ for all E-pucks, while the spacing policy parameters are selected as $r_{i}=0.1 \mathrm{~m}$ and $h_{i}=0.05 \mathrm{~s}$. The gains are selected in an iterative manner. Identical to the simulations, higher gains result in a faster convergence to the reference path, but a higher sensitivity to curvature changes. In the experiment, this is undesirable since a slight change in measurements, due to noise and sensor inaccuracy, could result in overcompensation. Both systems with the conventional and extended look-ahead are implemented, and the experiment results are presented in the next section to verify the simulation results. Since the E-puck has a velocity limitation $(13 \mathrm{~cm} / \mathrm{s})$, all experiments were performed at low velocity.

\section{B. Circular Path Scenario}

In this scenario, we define the initial position of the reference trajectory at $(0.5,0.1)$. Four E-pucks that are already in a platoon order are placed behind that initial position to ensure that they move forward. The reference trajectory itself acts as a reference only for the first E-puck, while the others use their preceding E-puck as a reference, i.e., E-puck 2 follows E-puck 1, E-puck 3 follows E-puck 2, and so on. The reference vehicle cruises in a straight trajectory with the velocity of $0.04 \mathrm{~m} / \mathrm{s}$ along global $x$-axis until it reaches $x=0.8 \mathrm{~m}$, and then maneuvers into a circular trajectory. The trajectories of all E-pucks with the conventional and extended look-ahead controllers are shown in Figs. 12 and 13,

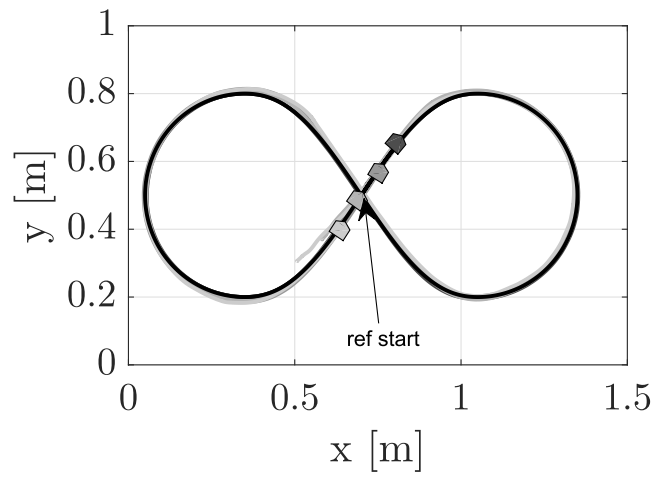

Fig. 17. Trajectory of E-puck $i$ (black-light gray: $i=1,2,3,4$ ) on an eight-shaped path with extended look-ahead.
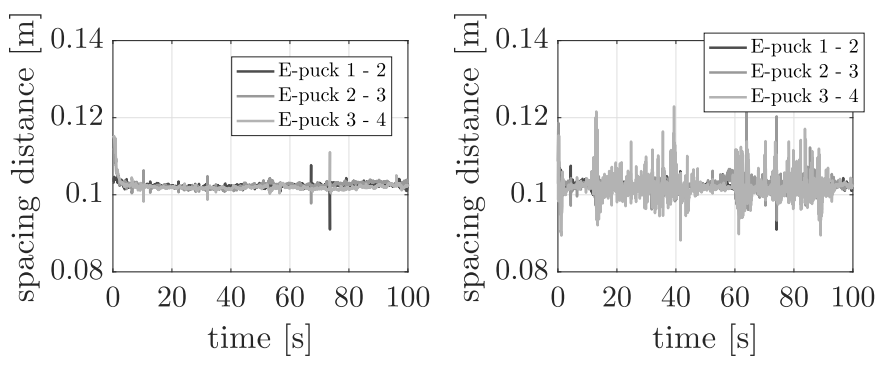

Fig. 18. Longitudinal spacing distance for the circular path scenario: conventional look-ahead (left) versus extended look-ahead (right).

respectively. As observed in both figures, on the straight trajectory all robots directly move to their desired position, subject to the spacing distance between robots. However, on the circular trajectory, the platoon with the conventional look-ahead approach suffers from the cutting-corner behavior, indicated by a smaller turning radius for each robot upstream. In contrast, the extended look-ahead controller shows satisfactory results, with all E-pucks driving closely to the reference trajectory. Moreover, the extended look-ahead controller also successfully maintains the longitudinal spacing distance between each E-puck at $0.1 \mathrm{~m}$ (see Fig. 14). It can be observed from Fig. 15 that the velocities of all E-pucks are nonzero. It should be noted that the differences between simulations and experiment regarding spacing distances and velocities at the start of the simulation and the experiment exist due to the different initial condition in simulation versus experiment. Moreover, there are also several factors that can affect the variation in the errors in experiment results: the stick-slip effects in the E-puck drive line, actuator delay, and the nondeterministic delay in the Bluetooth communication. In spite of these differences, it can be observed that our approach performs equally well in both simulations and experiments.

\section{Eight-Shaped Path Scenario}

For the second experiment, an eight-shaped path is used to confirm the robustness of the controller against a varying yaw rate. The reference trajectory starts at $(0.7,0.5)$ and the E-pucks are placed behind the reference's initial position to ensure that they move forward, i.e., $v_{i}>0$ for all $i$. The platoon moves with the same topology as the previous 

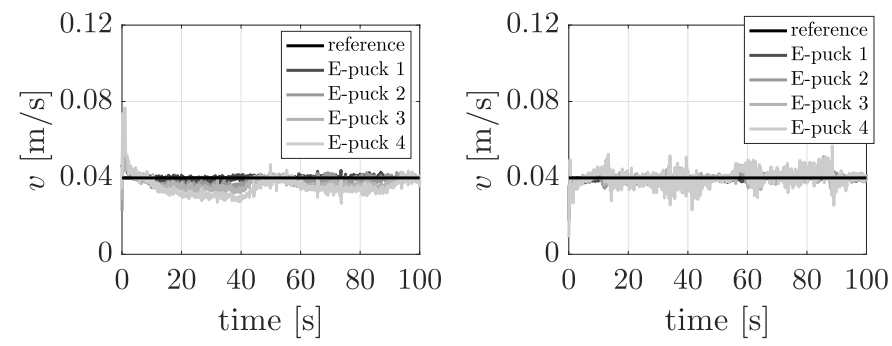

Fig. 19. Velocities of E-pucks for eight-shaped path: conventional look-ahead (left) versus extended look-ahead (right).

experiment. The first E-puck tracks the reference trajectory, while the others follow their preceding robot. As expected, the platoon with the conventional look-ahead controller tends to cut the corner, and the deviation in radius with the reference escalates in upstream direction (Fig. 16). The effectiveness of the extended look-ahead controller for the eight-shaped trajectory can be observed directly from Fig. 17. The radius deviation has been successfully compensated, thus resulting in a better tracking performance than the conventional lookahead. Due to the additional terms $\beta_{1, i}$ and $s_{a, i}$ in the extended look-ahead controller (35), the variation in the errors for the extended look-ahead is larger than the variation for the conventional look-ahead (see Fig. 18). Nevertheless, the measurement noise in the extended look-ahead is still small enough (less than $0.02 \mathrm{~m}$ ) such that the longitudinal spacing distance between each E-puck can still be maintained at $0.1 \mathrm{~m}$. It can also be observed that velocities of all E-pucks in this scenario are nonzero (Fig. 19).

\section{CONCLUSION}

Cooperative driving for a platoon of unicycles has been studied with the emphasis on cornering maneuvers. By using only the current position information of the preceding vehicle, the platoon suffers from a cutting-corner behavior, in which the follower vehicle has a smaller radius than the preceding vehicle. The difference in radius with respect to the first vehicle escalates upstream, thus affecting the tracking performance at cornering. To ensure a better performance, the error dynamics at cornering have been redefined. The redefined error dynamics involve the follower vehicle tracking the end of a vector extending from the preceding vehicle. This point can be viewed as a virtual vehicle which acts as the tracking objective for the follower vehicle. The effectiveness of the proposed approach has been successfully demonstrated in simulation, and validated by an experiment with a platoon consisting of four E-pucks. In this experiment, a vehicle following maneuver has been demonstrated on a circular path and an eight-shaped path. The designed extended look-ahead approach has been proven to compensate the cutting-corner behavior, while satisfying the spacing policy requirement between vehicles.

\section{APPENDIX A}

To prove Proposition 1, we first have to show that given the condition (12), the input (8) is well defined such that $z_{34, i}(t)$ remains bounded, using the following lemma.
Lemma 3: Let $z_{34, i}=\left[z_{3, i}, z_{4, i}\right]^{T}, \xi_{i}=\left[\xi_{1, i}, \xi_{2, i}\right]^{T}$, and consider the dynamics

$$
\dot{z}_{34, i}=-\left[\begin{array}{cc}
\frac{h_{i} v_{i}+r_{i} \cos ^{2} \theta_{i}}{h_{i}\left(r_{i}+h_{i} v_{i}\right)} & \frac{r_{i} \sin \theta_{i} \cos \theta_{i}}{h_{i}\left(r_{i}+h_{i} v_{i}\right)} \\
\frac{r_{i} \sin \theta_{i} \cos \theta_{i}}{h_{i}\left(r_{i}+h_{i} v_{i}\right)} & \frac{h_{i} v_{i}+r_{i} \sin ^{2} \theta_{i}}{h_{i}\left(r_{i}+h_{i} v_{i}\right)}
\end{array}\right] z_{34, i}+\xi_{i}
$$

with $v_{i}$ and $\theta_{i}$ as in (1), and input $\xi_{i}$ as a piecewise continuous function, bounded by $\left\|\xi_{i}\right\| \leq \xi_{i}^{\max }$. Let $\epsilon>0$ be given, and assume that $0<\epsilon<v_{i-1}^{\min } \leq v_{i-1}(t) \leq v_{i-1}^{\max }$.

1) If

$$
\left\|z_{34, i}(0)\right\| \leq\left(\frac{r_{i}}{\epsilon}+h_{i}\right) \xi_{i}^{\max } \leq v_{i-1}^{\min }-\epsilon
$$

then $v_{i}(t) \geq \epsilon$.

2) If, additionally,

$$
\lim _{t \rightarrow \infty}\left\|\xi_{i}(t)\right\|=0
$$

then $\lim _{t \rightarrow \infty}\left\|z_{34, i}(t)\right\|=0$.

Proof: Consider the set $\Omega_{c}=\left\{\left\|z_{34, i}\right\| \leq v_{i-1}^{\min }-\epsilon\right\}$. In $\Omega_{c}$, we have

$$
\begin{aligned}
\epsilon^{2} & \leq\left(v_{i-1}^{\min }-\left\|z_{34, i}\right\|\right)^{2} \leq\left(v_{i-1}-\sqrt{z_{3, i}^{2}+z_{4, i}^{2}}\right)^{2} \\
& =v_{i-1}^{2}-2 v_{i-1} \sqrt{z_{3, i}^{2}+z_{4, i}^{2}}+z_{3, i}^{2}+z_{4, i}^{2} .
\end{aligned}
$$

Define $c:=\left[\cos \theta_{i-1}, \sin \theta_{i-1}\right]^{T}$. With this particular definition of $c$, while using the Cauchy-Schwarz inequality, we have

$$
\begin{aligned}
2\left\|z_{34, i}\right\|\|c\| & \geq\left\|z_{34, i}+c\right\|^{2}-\left\|z_{34, i}\right\|^{2}-\|c\|^{2} \\
2 \sqrt{z_{3, i}^{2}+z_{4, i}^{2}} & \geq 2 z_{3, i} \cos \theta_{i-1}+2 z_{4, i} \sin \theta_{i-1}
\end{aligned}
$$

Substituting (46) into (45), while using (5c) and (5d), yields

$$
\begin{aligned}
\epsilon^{2} & \leq\left(v_{i-1} \cos \theta_{i-1}-z_{3, i}\right)^{2}+\left(v_{i-1} \sin \theta_{i-1}-z_{4, i}\right)^{2} \\
& \leq v_{i}^{2}
\end{aligned}
$$

which implies that inside $\Omega_{c}$ we have $v_{i}(t) \geq \epsilon>0$. Now we have to show that when a trajectory starts in $\Omega_{c}$, i.e., $\left\|z_{34, i}(0)\right\| \leq v_{i-1}^{\min }-\epsilon$, it will stay in $\Omega_{c}$ for all $t>0$. Consider to this end a Lyapunov function $V=z_{3, i}^{2}+z_{4, i}^{2}$. The derivative of $V$ along the trajectory of the system (42) is then given by

$$
\dot{V}=\phi+2 \xi_{1, i} z_{3, i}+2 \xi_{2, i} z_{4, i}
$$

with

$\phi:=-\frac{2 v_{i}}{r_{i}+h_{i} v_{i}}\left(z_{3, i}^{2}+z_{4, i}^{2}+\frac{r_{i}}{h_{i}}\left(z_{3, i} \cos \theta_{i}+z_{4, i} \sin \theta_{i}\right)^{2}\right)$.

Note that, since $v_{i} \geq \epsilon>0$, in the set $\Omega_{c}$,

$$
\phi \leq-\frac{2 v_{i}}{r_{i}+h_{i} v_{i}}\left(z_{3, i}^{2}+z_{4, i}^{2}\right)=-\frac{2 v_{i}}{r_{i}+h_{i} v_{i}}\left\|z_{34, i}\right\|^{2} .
$$

Moreover, by the Cauchy-Schwarz inequality, we have

$$
\begin{aligned}
\| \xi_{i} & +z_{34, i}\left\|^{2}-\right\| \xi_{i}\left\|^{2}-\right\| z_{34, i} \|^{2} \\
& \leq 2\left\|z_{34, i}\right\|\left\|\xi_{i}\right\| \\
2 \xi_{1, i} z_{3, i}+2 \xi_{2, i} z_{4, i} & \leq 2\left\|z_{34, i}\right\|\left\|\xi_{i}\right\| .
\end{aligned}
$$


Consequently,

$$
\begin{aligned}
\dot{V} \leq & -\frac{2 v_{i}}{r_{i}+h_{i} v_{i}}\left\|z_{34, i}\right\|^{2}+2\left\|z_{34, i}\right\|\left\|\xi_{i}\right\| \\
= & -\frac{v_{i}}{r_{i}+h_{i} v_{i}}\left\|z_{34, i}\right\|^{2}-\frac{v_{i}}{r_{i}+h_{i} v_{i}} \\
& \times\left(\left\|z_{34, i}\right\|-\frac{r_{i}+h_{i} v_{i}}{v_{i}}\left\|\xi_{i}\right\|\right)^{2} \\
& +\frac{r_{i}+h_{i} v_{i}}{v_{i}}\left\|\xi_{i}\right\|^{2} \\
\leq & -\frac{v_{i}}{r_{i}+h_{i} v_{i}}\left(\left\|z_{34, i}\right\|^{2}-\left(\frac{r_{i}+h_{i} v_{i}}{v_{i}} \xi_{i}^{\max }\right)^{2}\right)
\end{aligned}
$$

and it follows that the negative definiteness of $\dot{V}$ is determined by $v_{i}$ and $\left\|z_{34, i}\right\|$. Note that by (43) and by the fact that $v_{i} \geq \epsilon$, we have $\left(r_{i} / v_{i}+h_{i}\right) \xi_{i}^{\max } \leq\left(r_{i} / \epsilon+h_{i}\right) \xi_{i}^{\max } \leq v_{i-1}^{\min }-\epsilon$. Now let us consider two sets, $\Omega_{u}=\left\{\left\|z_{34, i}\right\|<\left(r_{i} / v_{i}+h_{i}\right) \xi_{i}^{\max }\right\}$ and $\Omega_{s}=\left\{\left(r_{i} / v_{i}+h_{i}\right) \xi_{i}^{\max } \leq\left\|z_{34, i}\right\| \leq v_{i-1}^{\min }-\epsilon\right\}$. Inside $\Omega_{u}$ [which is a subset of $\Omega_{c}$, according to condition (43)], we have $v_{i} \geq \epsilon>0$ and $\dot{V}>0$. Consequently, a trajectory starting in $\Omega_{u}$ will move in a direction of increasing $V$ until it reaches the lower bound $\left(r_{i} / v_{i}+h_{i}\right) \xi_{i}^{\max }$ of $\Omega_{s}$. Inside $\Omega_{s}$ (which is also a subset of $\Omega_{c}$ ), $v_{i} \geq \epsilon>0$ and it follows from (49) that $\dot{V}$ is negative in $\Omega_{s}$. Hence, a trajectory that starts in $\Omega_{s}$ will also converge to the lower bound of $\Omega_{s}$. Since $v_{i} \geq \epsilon$ implies that $\left(r_{i} / v_{i}+h_{i}\right) \xi_{i}^{\max } \leq\left(r_{i} / \epsilon+h_{i}\right) \xi_{i}^{\max }$, we can conclude that for $\left\|z_{34, i}(0)\right\| \leq\left(r_{i} / \epsilon+h_{i}\right) \xi_{i}^{\max } \leq v_{i-1}^{\min }-\epsilon$ we have $\left\|z_{34, i}(t)\right\| \leq\left(r_{i} / \epsilon+h_{i}\right) \xi_{i}^{\max } \leq v_{i-1}^{\min }-\epsilon$.

Now we want to prove the second statement of Lemma 3. Let $\lim _{t \rightarrow \infty}\left\|\xi_{i}(t)\right\|=0$. Since $\left\|z_{34, i}(t)\right\|$ converges to $\left(r_{i} / v_{i}+\right.$ $\left.h_{i}\right) \xi_{i}$ (which follows from (49) in the previous analysis), we have $\lim _{t \rightarrow \infty}\left\|z_{34, i}(t)\right\|=0$.

To conclude the proof, we have to show that $\xi_{i}$ is bounded and converges to zero, given the condition (13). From (10), it can be observed that the boundedness of $\xi_{i}$ depends on the boundedness of $H_{i-1}\left[a_{i-1}, \omega_{i-1}\right]^{T}$ [which is given by (12)], boundedness of $G_{i}$, and the initial condition $z_{12, i}(0)$. From (11), it can be observed that the Frobenius norm of $G_{i}$ is bounded by $\left\|G_{i}\right\|_{\mathrm{F}} \leq 1 / h_{i}$. By having sufficiently small initial velocity error $z_{34, i}(0)$, we can guarantee that all the future trajectories of $z_{34, i}(t)$ will be in the set $\Omega_{s}$ in which the velocity $v_{i}$ is always positive, thus the input (8) is well defined. Since the input is well defined, then we also have $\lim _{t \rightarrow \infty}\left\|z_{12, i}(t)\right\|=0$. In addition, under the condition (13) and sufficiently small initial position error $z_{12, i}(0)$, from (10) we have that $\xi_{i}$ is bounded and converges to zero as $t \rightarrow \infty$. Therefore, by the second statement of Lemma 3, it can be concluded that $\lim _{t \rightarrow \infty}\left\|z_{34, i}(t)\right\|=0$.

\section{APPENDIX B}

First, we need to study the internal dynamics (38b). By substituting $H_{i-1}, \beta_{1, i}, \beta_{2, i}, \Gamma_{34, i}, \Gamma_{12, i}^{-1}$, separating the translational and rotational dynamics, and noting that $\omega_{i-1}=$ $v_{i-1} \kappa_{i-1}$, we are able to rewrite (38b) into

$$
\left[\begin{array}{c}
\dot{z}_{3, i} \\
\dot{z}_{4, i}
\end{array}\right]=-G_{i}\left[\begin{array}{l}
z_{3, i} \\
z_{4, i}
\end{array}\right]-\kappa_{i-1}\left(Q_{i}\left[\begin{array}{c}
z_{3, i} \\
z_{4, i}
\end{array}\right]-f_{v, i}\right)+\zeta_{i}
$$

where

$$
\begin{aligned}
& f_{v, i}=\left[\begin{array}{c}
-v_{i-1} \sin \theta_{i-1} \\
v_{i-1} \cos \theta_{i-1}
\end{array}\right] v_{i-1}-\left[\begin{array}{c}
-v_{i} \sin \left(\theta_{i}+\alpha\right) \\
v_{i} \cos \left(\theta_{i}+\alpha\right)
\end{array}\right] v_{i} \\
& \zeta_{i}=h_{\kappa, i} \dot{\kappa}_{i-1}+\left[\begin{array}{c}
\cos \theta_{i-1} \\
\sin \theta_{i-1}
\end{array}\right] a_{i-1}-\Gamma_{34, i} \Gamma_{12, i}^{-1}\left[\begin{array}{l}
k_{1} z_{1, i} \\
k_{2} z_{2, i}
\end{array}\right] \\
& h_{\kappa, i}=v_{i}\left(r+h_{i} v_{i}\right) \cos ^{2} \alpha_{i}\left[\begin{array}{c}
\sin \left(\theta_{i}+\alpha_{i}\right) \\
-\cos \left(\theta_{i}+\alpha_{i}\right)
\end{array}\right] \\
& +\frac{\cos \alpha_{i}-1}{\kappa_{i-1}^{2}} \Gamma_{34} \Gamma_{12}^{-1}\left[\begin{array}{c}
\sin \theta_{i-1} \\
-\cos \theta_{i-1}
\end{array}\right] \\
& Q_{i}=\frac{r_{i}+h_{i} v_{i}}{\mu_{i}}\left[\begin{array}{ll}
q_{11} & q_{12} \\
q_{21} & q_{22}
\end{array}\right] \\
& q_{11}=\cos \alpha_{i} \sin \left(\theta_{i-1}-\theta_{i}\right)\left(r_{i} \cos ^{2} \theta_{i}+h_{i} v_{i}\right) \\
& +h_{i} v_{i} \sin \left(\theta_{i}+\alpha_{i}\right)\left[\cos \theta_{i-1}-\cos \alpha_{i} \cos \theta_{i}\right] \\
& -r_{i} \sin \theta_{i} \cos \theta_{i} \\
& q_{12}=\cos \alpha_{i} \sin \left(\theta_{i-1}-\theta_{i}\right)\left(r_{i} \sin \theta_{i} \cos \theta_{i}\right) \\
& h_{i} v_{i} \sin \left(\theta_{i}+\alpha_{i}\right)\left[-\sin \theta_{i-1}+\cos \alpha \sin \theta_{i}\right] \\
& -r_{i} \sin ^{2} \theta_{i}-h_{i} v_{i} \\
& q_{21}=\cos \alpha_{i} \sin \left(\theta_{i-1}-\theta_{i}\right)\left(r_{i} \sin \theta_{i} \cos \theta_{i}\right) \\
& -h_{i} v_{i} \cos \left(\theta_{i}+\alpha_{i}\right)\left[\cos \theta_{i-1}-\cos \alpha_{i} \cos \theta_{i}\right] \\
& +r_{i} \cos ^{2} \theta_{i}+h_{i} v_{i} \\
& q_{22}=\cos \alpha_{i} \sin \left(\theta_{i-1}-\theta_{i}\right)\left(r_{i} \sin ^{2} \theta_{i}+h_{i} v_{i}\right) \\
& +h_{i} v_{i} \cos \left(\theta_{i}+\alpha_{i}\right)\left[-\sin \theta_{i-1}+\cos \alpha_{i} \sin \theta_{i}\right] \\
& +r_{i} \sin \theta_{i} \cos \theta_{i}
\end{aligned}
$$

with $\mu_{i}$ as in (37), and $G_{i}$ as in (11). It can be observed that if the platoon maneuvers on a straight path (i.e., $\kappa_{i-1}=0$ and $\omega_{i-1}=0$ ), the subsystem (50) reduces to $(9 \mathrm{~b})$, thus for tracking a straight path we can again use Proposition 1. The term $\zeta_{i}$ can be considered as external inputs that are decaying to zero. Given that $\kappa_{i-1}$ is bounded by (39) and the condition (40) is satisfied, we first prove that the subsystem (50) is stable by the following lemma.

Lemma 4: Let $z_{34, i}=\left[z_{3, i}, z_{4, i}\right]^{T}, \zeta_{i}=\left[\zeta_{1, i}, \zeta_{2, i}\right]^{T}$, and consider the dynamics (50) where $\kappa_{i-1}$ is bounded by (39) and input $\zeta_{i}$ is a continuous function, bounded by $\left\|\zeta_{i}\right\| \leq \zeta_{i}^{\max }$. Let $\epsilon>0$ be given, and assume that $0<\epsilon<v_{i-1}^{\min } \leq v_{i-1}(t) \leq$ $v_{i-1}^{\max }$.

1) If

$$
\left\|z_{34, i}(0)\right\| \leq 2\left(\frac{r_{i}}{\epsilon}+h_{i}\right) \zeta_{i}^{\max } \leq v_{i-1}^{\min }-\epsilon
$$

then $v_{i}(t) \geq 0$.

2) If, additionally,

$$
\lim _{t \rightarrow \infty}\left\|\zeta_{i}(t)\right\|=0
$$

then $\lim _{t \rightarrow \infty}\left\|z_{34, i}(t)\right\|=0$.

Proof: Let us consider the set $\Omega_{c}=\left\{\left\|z_{34, i}\right\| \leq v_{i-1}^{\min }-\epsilon\right\}$, with $\epsilon>0$. Inside $\Omega_{c}$, we have

$$
\begin{aligned}
\epsilon^{2} & \leq\left(v_{i-1}^{\min }-\left\|z_{34, i}\right\|\right)^{2} \leq\left(v_{i-1}-\sqrt{z_{3, i}^{2}+z_{4, i}^{2}}\right)^{2} \\
& =v_{i-1}^{2}-2 v_{i-1} \sqrt{z_{3, i}^{2}+z_{4, i}^{2}}+z_{3, i}^{2}+z_{4, i}^{2} \\
& \leq\left(v_{i-1} \cos \theta_{i-1}-z_{3, i}\right)^{2}+\left(v_{i-1} \sin \theta_{i-1}-z_{4, i}\right)^{2} \\
& =v_{i}^{2} .
\end{aligned}
$$


Moreover, it directly follows from (19c) and (19d) that:

$$
\begin{aligned}
v_{i}^{2}= & \left(v_{i-1} \cos \theta_{i-1}-z_{3, i}\right)^{2}+\left(v_{i-1} \sin \theta_{i-1}-z_{4, i}\right)^{2} \\
= & v_{i-1}^{2}-2 v_{i-1} z_{3, i} \cos \theta_{i-1}-2 v_{i-1} z_{4, i} \sin \theta_{i-1}+\left\|z_{34, i}\right\|^{2} \\
\leq & v_{i-1}^{2}+v_{i-1}^{2} \cos ^{2} \theta_{i-1}+z_{3, i}^{2} \\
& +v_{i-1}^{2} \sin ^{2} \theta_{i-1}+z_{4, i}^{2}+\left\|z_{34, i}\right\|^{2} \\
= & 2 v_{i-1}^{2}+2\left\|z_{34, i}\right\|^{2} \leq 2\left(v_{i-1}+\left\|z_{34, i}\right\|\right)^{2} .
\end{aligned}
$$

As a result from (57) and (58), $v_{i}$ is bounded by

$$
0<\epsilon \leq v_{i} \leq \sqrt{2}\left(v_{i-1}^{\max }+v_{i-1}^{\min }-\epsilon\right)
$$

in $\Omega_{c}$. Now consider a quadratic Lyapunov function $V=z_{3, i}^{2}+$ $z_{4, i}^{2}$. By using $(19 \mathrm{c}, 19 \mathrm{~d})$, we can rewrite $f_{v, i}$ in (51) as

$$
f_{v, i}=\left[\begin{array}{c}
-v_{i-1} \sin \theta_{i-1} \\
v_{i-1} \cos \theta_{i-1}
\end{array}\right]\left(v_{i-1}-v_{i}\right)+v_{i}\left[\begin{array}{c}
-z_{4, i} \\
z_{3, i}
\end{array}\right]
$$

such that the derivative of $V$ along the trajectory (50) is given by

$$
\begin{aligned}
\dot{V}= & -2\left[\begin{array}{l}
z_{3, i} \\
z_{4, i}
\end{array}\right]^{T} G_{i}\left[\begin{array}{l}
z_{3, i} \\
z_{4, i}
\end{array}\right]-2 \kappa_{i-1}\left[\begin{array}{l}
z_{3, i} \\
z_{4, i}
\end{array}\right]^{T} Q_{i}\left[\begin{array}{l}
z_{3, i} \\
z_{4, i}
\end{array}\right] \\
& +2 \kappa_{i-1}\left(v_{i-1}-v_{i}\right)\left[\begin{array}{l}
z_{3, i} \\
z_{4, i}
\end{array}\right]^{T}\left[\begin{array}{c}
-v_{i-1} \sin \theta_{i-1} \\
v_{i-1} \cos \theta_{i-1}
\end{array}\right] \\
& +2\left[\begin{array}{l}
\zeta_{i, i} \\
\zeta_{i, 2}
\end{array}\right]^{T}\left[\begin{array}{l}
z_{3, i} \\
z_{4, i}
\end{array}\right]
\end{aligned}
$$

By substituting $G_{i}$ and $Q_{i}$ into (61) and by noting that $\sin \alpha_{i}=$ $\kappa_{i-1}\left(r_{i}+h_{i} v_{i}\right) \cos \alpha_{i}$ [which directly follows from (21) and (22)], we can rewrite $\dot{V}$ as

$$
\begin{aligned}
\dot{V} & -\frac{2 v_{i}}{\gamma_{i}\left(r_{i}+h_{i} v_{i}\right)}\left(z_{3, i}^{2}+z_{4, i}^{2}+\frac{r_{i}}{h_{i} v_{i}}\left(z_{3, i} \cos \theta_{i}+z_{4, i} \sin \theta_{i}\right)^{2}\right) \\
& +\frac{2 r_{i} \kappa_{i-1}}{\gamma_{i} h_{i}}\left(z_{3, i} \sin \theta_{i}-z_{4, i} \cos \theta_{i}\right)\left(z_{3, i} \cos \theta_{i}+z_{4, i} \sin \theta_{i}\right) \\
& -\frac{2 v_{i} \kappa_{i-1}}{\gamma_{i}} f_{34, i}\left(z_{3, i} \cos \theta_{i-1}+z_{4, i} \sin \theta_{i-1}\right) \\
& +\frac{2 v_{i} \kappa_{i-1} \cos \alpha_{i}}{\gamma_{i}} f_{34, i}\left(z_{3, i} \cos \theta_{i}+z_{4, i} \sin \theta_{i}\right) \\
& +2 \kappa_{i-1} v_{i-1}\left(v_{i-1}-v_{i}\right)\left(-z_{3, i} \sin \theta_{i-1}+z_{4, i} \cos \theta_{i-1}\right) \\
& +2 \zeta_{1, i} z_{3, i}+2 \zeta_{2, i} z_{4, i}
\end{aligned}
$$

where

$$
\begin{aligned}
f_{34, i} & =\left(z_{3, i} \sin \left(\theta_{i}+\alpha_{i}\right)-z_{4, i} \cos \left(\theta_{i}+\alpha_{i}\right)\right) \\
\gamma_{i} & =1-\sin \alpha_{i} \sin \left(\theta_{i-1}-\theta_{i}\right) .
\end{aligned}
$$

Note that we can also apply the Cauchy-Schwarz inequality [in the same manner as (46)] to all terms in (62) that are dependent on $z_{3, i}$ and $z_{4, i}$. As a result, those terms are always less than or equal to $\left\|z_{34, i}\right\|$. Moreover, by applying the inequality to (19c) and (19d), we also have $v_{i-1}-v_{i} \leq\left\|z_{34, i}\right\|$. Since $\gamma_{i} \leq 2$ by definition (64), it follows from (49) that:

$$
\begin{aligned}
\dot{V} \leq & -\left(\frac{v_{i}}{r_{i}+h_{i} v_{i}}\right)\left\|z_{34, i}\right\|^{2}-\kappa_{i-1}\left(-\frac{r_{i}}{h_{i}}\right)\left\|z_{34, i}\right\|^{2} \\
& -v_{i} \kappa_{i-1}\left\|z_{34, i}\right\|^{2}+v_{i} \kappa_{i-1} \cos \alpha_{i}\left\|z_{34, i}\right\|^{2} \\
& +2 \kappa_{i-1} v_{i-1}\left\|z_{34, i}\right\|^{2}+2\left\|z_{34, i}\right\|\left\|\zeta_{i}\right\|
\end{aligned}
$$

and we need to show that $\dot{V} \leq 0$. Let us denote

$$
\Delta_{i}=\frac{v_{i}}{2\left(r_{i}+h_{i} v_{i}\right)}+\kappa_{i-1}\left(-\frac{r_{i}}{h_{i}}+v_{i}\left(1-\cos \alpha_{i}\right)-2 v_{i-1}\right)
$$

such that we can rewrite (65) as

$$
\begin{aligned}
\dot{V} \leq & -\Delta_{i}\left\|z_{34, i}\right\|^{2}-\frac{v_{i}}{2\left(r_{i}+h_{i} v_{i}\right)}\left\|z_{34, i}\right\|^{2}+2\left\|z_{34, i}\right\|\left\|\zeta_{i}\right\| \\
= & -\Delta_{i}\left\|z_{34, i}\right\|^{2}-\frac{v_{i}}{4\left(r_{i}+h_{i} v_{i}\right)}\left\|z_{34, i}\right\|^{2}+\frac{4\left(r_{i}+h_{i} v_{i}\right)}{v_{i}}\left\|\zeta_{i}\right\|^{2} \\
& -\frac{v_{i}}{4\left(r_{i}+h_{i} v_{i}\right)}\left(\left\|z_{34, i}\right\|-\frac{4\left(r_{i}+h_{i} v_{i}\right)}{v_{i}}\left\|\zeta_{i}\right\|\right)^{2} \\
\leq & -\Delta_{i}\left\|z_{34, i}\right\|^{2}-\frac{v_{i}}{4\left(r_{i}+h_{i} v_{i}\right)} \\
& \times\left(\left\|z_{34, i}\right\|^{2}-\left(\frac{4\left(r_{i}+h_{i} v_{i}\right)}{v_{i}} \zeta_{i}^{\max }\right)^{2}\right)
\end{aligned}
$$

and it follows that the negative definiteness of $\dot{V}$ is determined by $v_{i},\left\|z_{34, i}\right\|$, and $\Delta_{i}$. First we want to analyze the term $\Delta_{i}$. Note that by using (22), and by using a Taylor expansion we can bound the term $\left(1-\cos \alpha_{i}\right)$ by

$$
\begin{aligned}
1-\cos \alpha_{i} & =\frac{\sqrt{1+\kappa_{i-1}^{2}\left(r_{i}+h_{i} v_{i}\right)^{2}}-1}{\sqrt{1+\kappa_{i-1}^{2}\left(r_{i}+h_{i} v_{i}\right)^{2}}} \\
& \leq \frac{1}{2} \kappa_{i-1}^{2}\left(r_{i}+h_{i} v_{i}\right)^{2} .
\end{aligned}
$$

Moreover, due to the fact that $v_{i-1} \geq v_{i-1}^{\min }>0$, it follows from (66) that:

$v_{i}-2\left|\kappa_{i-1}\right|\left(r_{i}+h_{i} v_{i}\right)\left(-\frac{r_{i}}{h_{i}}+\frac{1}{2} v_{i}\left|\kappa_{i-1}\right|^{2}\left(r_{i}+h_{i} v_{i}\right)^{2}\right)>0$

is a sufficient condition for the first term in (67) to be negative. Using condition (39) and the bound of $v_{i}$ from (59), we have

$$
\left|\kappa_{i-1}\right| \leq \frac{1}{r_{i}+h_{i} \sqrt{2}\left(v_{i-1}^{\max }+v_{i-1}^{\min }-\epsilon\right)} \leq \frac{1}{r_{i}+h_{i} v_{i}}
$$

where the second inequality follows from the upper bound of $v_{i}$ in (59). By substituting (70) into (69), we obtain

$$
v_{i}-2\left(-\frac{r_{i}}{h_{i}}+\frac{1}{2} v_{i}\right)=\frac{2 r_{i}}{h_{i}}>0
$$

from which we can conclude that $\Delta_{i}$ is positive for all $\kappa_{i-1}$ satisfying (39). Now, we want to show that the second term of (67) is negative. Note by (55) and by the fact that $v_{i} \geq \epsilon$ from (59), we have $2\left(r_{i} / v_{i}+h_{i}\right) \zeta_{i}^{\max } \leq 2\left(r_{i} / \epsilon+h_{i}\right) \zeta_{i}^{\max } \leq$ $v_{i-1}^{\min }-\epsilon$. Now let us consider two subsets of $\Omega_{c}$, denote by $\Omega_{u}=\left\{\left\|z_{34, i}\right\|<2\left(r_{i} / v_{i}+h_{i}\right) \zeta_{i}^{\max }\right\}$ and $\Omega_{s}=\left\{2\left(r_{i} / v_{i}+\right.\right.$ $\left.\left.h_{i}\right) \zeta_{i}^{\max } \leq\left\|z_{34, i}\right\| \leq v_{i-1}^{\min }-\epsilon\right\}$. By using the same reasoning as Lemma 3, we can also conclude that for $\left\|z_{34, i}(0)\right\| \leq 2\left(r_{i} / \epsilon+\right.$ $\left.h_{i}\right) \zeta_{i}^{\max } \leq v_{i-1}^{\min }-\epsilon$ we have $\left\|z_{34, i}(t)\right\| \leq 2\left(r_{i} / \epsilon+h_{i}\right) \zeta_{i}^{\max } \leq$ $v_{i-1}^{\min }-\epsilon$, given that $\kappa_{i-1}$ is strictly bounded by (39).

The second statement of Lemma 4 can be proven directly using the same reasoning as the one in Lemma 3. 
From Lemma 4, we have the result that the subsystem (50) is stable, under the condition that $\kappa_{i-1}$ is bounded, and also under the condition that $\zeta_{i}$ is bounded by

$$
\zeta_{i}(t) \leq \frac{v_{i-1}^{\min }-\epsilon}{2\left(\frac{r_{i}}{\epsilon}+h_{i}\right)}
$$

with $\zeta_{i}$ as in (52), and can be considered as external inputs that are decaying to zero. To conclude the stability proof of this system, we need to check the boundedness of $\Gamma_{34, i} \Gamma_{12, i}^{-1}$ and $h_{\kappa, i}$. From (21) and (22), we have

$$
\cos ^{2} \alpha_{i}=\frac{\sin \alpha_{i} \cos \alpha_{i}}{\kappa_{i-1}\left(r_{i}+h_{i} v_{i}\right)} .
$$

Substituting (72) into $\delta_{i}$ in (31) and taking (30) into account, we eventually obtain $\Gamma_{34, i} \Gamma_{12, i}^{-1}$ as

$$
\Gamma_{34} \Gamma_{12}^{-1}=\frac{1}{\mu_{i}}\left[\begin{array}{ll}
\gamma_{11} & \gamma_{12} \\
\gamma_{21} & \gamma_{22}
\end{array}\right]
$$

with

$$
\begin{aligned}
\gamma_{11} & =\left(r_{i}+h_{i} v_{i}\right) \cos \theta_{i} \cos \left(\theta_{i}+\alpha_{i}\right)+h_{i} v_{i} \sin \left(\theta_{i}+\alpha_{i}\right) p_{1} \\
\gamma_{12} & =\left(r_{i}+h_{i} v_{i}\right) \sin \theta_{i} \cos \left(\theta_{i}+\alpha_{i}\right)-h_{i} v_{i} \sin \left(\theta_{i}+\alpha_{i}\right) p_{2} \\
\gamma_{21} & =\left(r_{i}+h_{i} v_{i}\right) \cos \theta_{i} \sin \left(\theta_{i}+\alpha_{i}\right)-h_{i} v_{i} \cos \left(\theta_{i}+\alpha_{i}\right) p_{1} \\
\gamma_{22} & =\left(r_{i}+h_{i} v_{i}\right) \sin \theta_{i} \sin \left(\theta_{i}+\alpha_{i}\right)+h_{i} v_{i} \cos \left(\theta_{i}+\alpha_{i}\right) p_{2} \\
p_{1} & =\sin \theta_{i}+\sin \alpha_{i} \cos \theta_{i-1}-\sin \alpha_{i} \cos \alpha_{i} \cos \theta_{i} \\
p_{2} & =\cos \theta_{i}-\sin \alpha_{i} \sin \theta_{i-1}+\sin \alpha_{i} \cos \alpha_{i} \sin \theta_{i}
\end{aligned}
$$

and $\mu_{i}$ as in (37). Applying the fact that $\left(1-\sin \alpha_{i} \sin \left(\theta_{i-1}-\right.\right.$ $\left.\left.\theta_{i}\right)\right) \leq 2$ into $\mu_{i}$, we have

$$
\left\|\Gamma_{34, i} \Gamma_{12, i}^{-1}\right\|_{2} \leq\left\|\Gamma_{34, i} \Gamma_{12, i}^{-1}\right\|_{\mathrm{F}} \leq \frac{2}{h_{i}},
$$

where $\|\cdot\|_{\mathrm{F}}$ is the Frobenius norm. Thus, $\Gamma_{34, i} \Gamma_{12, i}^{-1}$ is bounded. Now, we want to show that $h_{\kappa, i}$ is also bounded. Note that by substituting (22) and (68) into (53), we can rewrite $h_{\kappa, i}$ as

$$
\begin{aligned}
h_{\kappa, i} \leq & \frac{v_{i}\left(r_{i}+h_{i} v_{i}\right)}{1+\kappa_{i-1}^{2}\left(r_{i}+h_{i} v_{i}\right)^{2}}\left[\begin{array}{c}
\sin \left(\theta_{i}+\alpha_{i}\right) \\
-\cos \left(\theta_{i}+\alpha_{i}\right)
\end{array}\right] \\
& +\frac{1}{2}\left(r_{i}+h_{i} v_{i}\right)^{2} \Gamma_{34} \Gamma_{12}^{-1}\left[\begin{array}{c}
\sin \theta_{i-1} \\
-\cos \theta_{i-1}
\end{array}\right] .
\end{aligned}
$$

By substituting (74) into (75), for a trajectory starting in $\Omega_{c}$ [which follows from (59)] we obtain

$$
\begin{aligned}
\left\|h_{\kappa, i}\right\|_{2} & \leq v_{i}\left(r_{i}+h_{i} v_{i}\right)-\frac{1}{h}\left(r_{i}+h_{i} v_{i}\right)^{2} \\
& \leq \frac{r_{i}^{2}}{h_{i}}+h_{i} \sqrt{2}\left(v_{i-1}^{\max }+v_{i-1}^{\min }-\epsilon\right)=h_{\kappa, i}^{\max } .
\end{aligned}
$$

Since we have that $\dot{\kappa}_{i-1}, a_{i-1}$ and $z_{12, i}$ converge to zero as $t \rightarrow \infty$, thus we have $\lim _{t \rightarrow \infty}\left\|z_{34, i}(t)\right\|=0$.

\section{REFERENCES}

[1] A. Vahidi and A. Eskandarian, "Research advances in intelligent collision avoidance and adaptive cruise control," IEEE Trans. Intell. Transp. Syst., vol. 4, no. 3, pp. 143-153, Sep. 2003.

[2] G. Naus, R. Vugts, J. Ploeg, R. van de Molengraft, and M. Steinbuch, "Cooperative adaptive cruise control, design and experiments," in Proc. Amer. Control Conf., 2010, vol. 1, no. 1, pp. 6145-6150.
[3] J. Ploeg, B. T. M. Scheepers, E. van Nunen, N. van de Wouw, and $\mathrm{H}$. Nijmeijer, "Design and experimental evaluation of cooperative adaptive cruise control," in Proc. 14th Int. IEEE Conf. Intell. Transp. Syst. (ITSC), Oct. 2011, pp. 260-265.

[4] S. E. Shladover, "Automated vehicles for highway operations (automated highway systems)," Proc. Inst. Mech. Eng., I, J. Syst. Control Eng., vol. 219, no. 1, pp. 53-75, Nov. 2005.

[5] A. A. Alam, A. Gattami, and K. H. Johansson, "An experimental study on the fuel reduction potential of heavy duty vehicle platooning," in Proc. 13th Int. IEEE Conf. Intell. Transp. Syst., Sep. 2010, pp. 306-311.

[6] R. Rajamani, H.-S. Tan, B. K. Law, and W.-B. Zhang, "Demonstration of integrated longitudinal and lateral control for the operation of automated vehicles in platoons," IEEE Trans. Control Syst. Technol., vol. 8, no. 4, pp. 695-708, Jul. 2000.

[7] S. Sheikholeslam and C. A. Desoer, "Combined longitudinal and lateral control of a platoon of vehicles," in Proc. Amer. Control Conf., vol. 1, Jun. 1992, pp. 1763-1767.

[8] E. Lim and J. Hedrick, "Lateral and longitudinal vehicle control coupling for automated vehicle operation," in Proc. Amer. Control Conf., vol. 5, Jun. 1999, pp. 3676-3680.

[9] Ö. Tunçer, L. Güvenç, F. Coșkun, and E. Karsligil, "Vision based lane keeping assistance control triggered by a driver inattention monitor," in Proc. IEEE Int. Conf. Syst., Man, Cybern., Oct. 2010, pp. 289-297.

[10] R. Kianfar, M. Ali, P. Falcone, and J. Fredriksson, "Combined longitudinal and lateral control design for string stable vehicle platooning within a designated lane," in Proc. IEEE 17th Int. Conf. Intell. Transp. Syst. (ITSC), Oct. 2014, pp. 1003-1008.

[11] S. Solyom, A. Idelchi, and B. B. Salamah, "Lateral control of vehicle platoons," in Proc. IEEE Int. Conf. Syst., Man, Cybern., Oct. 2013, pp. 4561-4565.

[12] P. Petrov, "A mathematical model for control of an autonomous vehicle convoy," WSEAS Trans. Syst. Control, vol. 3, no. 9, pp. 835-848, Mar. 2008.

[13] J. Ploeg, N. van de Wouw, and H. Nijmeijer, "Lp string stability of cascaded systems: Application to vehicle platooning," IEEE Trans. Control Syst. Technol., vol. 22, no. 2, pp. 786-793, Mar. 2014.

[14] S. K. Gehrig and F. J. Stein, "A trajectory-based approach for the lateral control of car following systems," in Proc. IEEE Int. Conf. Syst., Man, Cybern., vol. 4, Oct. 1998, pp. 3596-3601.

[15] M. Pham and D. Wang, "Unified control design for autonomous carlike vehicle tracking maneuvers," in Autonomous Mobile Robots. Boca Raton, FL, USA: Taylor \& Francis, 2006, pp. 295-329.

[16] A. Bayuwindra, O. L. Aakre, J. Ploeg, and H. Nijmeijer, "Combined lateral and longitudinal CACC for a unicycle-type platoon," in Proc. IEEE Intell. Vehicles Symp., Jun. 2016, pp. 527-532, no. 4.

[17] J. Caarls, "Pose estimation for mobile devices and augmented reality," Ph.D. dissertation, Fac. Appl. Sci., Delft Univ. Technol., Delft, The Netherlands, 2009.

[18] F. Mondada and M. Bonani. (2007). E-Puck Education Robot. [Online]. Available: http://www.e-puck.org

[19] T. H. A. van den Broek, N. van de Wouw, and H. Nijmeijer, "Formation control of unicycle mobile robots: A virtual structure approach," in Proc. Joint 48th IEEE Conf. Decis. Control 28th Chin. Control Conf., Dec. 2009, pp. 8328-8333.

[20] A. Morales and H. Nijmeijer, "Merging strategy for vehicles by applying cooperative tracking control," IEEE Trans. Intell. Transp. Syst., vol. 17, no. 12, pp. 3423-3433, Dec. 2016.

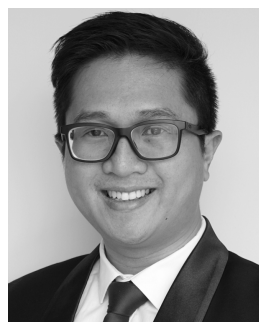

Anggera Bayuwindra received the B.Sc. degree in electrical engineering and the M.Sc. degree in electrical engineering (specializing in control and intelligent system) from the Bandung Institute of Technology, Bandung, Indonesia, in 2006 and 2012, respectively. He is currently pursuing the Ph.D. degree with the Department of Mechanical Engineering, Eindhoven University of Technology, Eindhoven, The Netherlands.

He was an Engineer with Infineon Technologies, Batam, Indonesia. His current research interests include nonlinear control system design, in particular the integrated longitudinal and lateral control for cooperative and automated vehicles. 


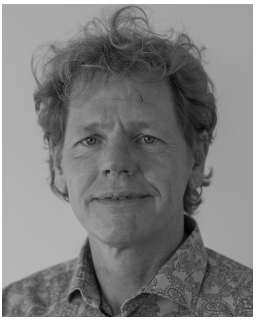

Jeroen Ploeg received the M.Sc. degree in mechanical engineering from the Delft University of Technology, Delft, The Netherlands, in 1988, and the Ph.D. degree in mechanical engineering on the control of vehicle platoons from the Eindhoven University of Technology, Eindhoven, The Netherlands, in 2014 .

From 1989 to 1999 , he was with Tata Steel, IJmuiden, The Netherlands, where he was involved in the development and implementation of dynamic process control systems for large-scale industrial plants. From 1999 to 2017, he was as a Principal Scientist with TNO, Helmond, The Netherlands, where he was involved in the field of vehicle automation and road safety assessment. Since 2017, he holds a position of part-time Associate Professor with the Department of Mechanical Engineering, Eindhoven University of Technology, Eindhoven, The Netherlands. $\mathrm{He}$ is currently with 2 getthere, Utrecht, The Netherlands, where he leads the research and development activities in the field of cooperative automated driving for automated transit systems, in particular platooning. His current research interests include control system design for cooperative and automated vehicles, in particular string stability of vehicle platoons, design of interaction protocols for complex driving scenarios, and motion control of wheeled mobile robots.

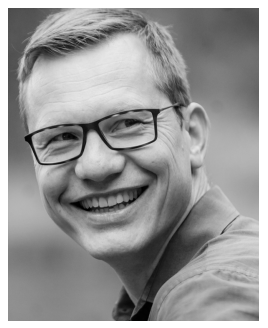

Erjen Lefeber received the M.Sc. degree in applied mathematics and the Ph.D. degree in the subject of tracking control of nonlinear mechanical systems from the University of Twente, Enschede, The Netherlands, in 1996 and 2000, respectively.

Since 2000, he has been an Assistant Professor with the Department of Mechanical Engineering, Eindhoven University of Technology, Eindhoven, The Netherlands, where he was involved in the modeling and control of manufacturing systems from 2000 to 2015 , and then he joined the Dynamics and Control Group in 2015. His current research interests include nonlinear control theory, in particular the control of drones and the control of platooning vehicles.

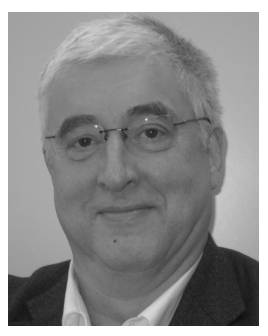

Henk Nijmeijer (F'00) is currently a Full Professor with the Eindhoven University of Technology, Eindhoven, The Netherlands, and also the Chair of the Dynamics and Control Group. He has authored or coauthored a large number of journal and conference papers and several books.

Prof. Nijmeijer has been an IFAC Council Member since 2011. He is a corresponding member of the Mexican Academy of sciences. He was a recipient of the 2015 IEEE Control Systems Technology Award and the IEE Heaviside Premium. He is appointed honorary knight of the Golden Feedback Loop (NTNU) in 2011. He was the Scientific Director of the Dutch Institute of Systems and Control in 2015. $\mathrm{He}$ is or was at the editorial board of numerous journals. He is an editor of the Communications in Nonlinear Science and Numerical Simulations. 